\title{
Interacting crumpled manifolds
}

\author{
Henryk A Pinnow ${ }^{1}$ and Kay Jörg Wiese ${ }^{1,2}$ \\ ${ }^{1}$ Fachbereich Physik, Universität Essen, 45117 Essen, Germany \\ 2 ITP, Kohn Hall, University of California at Santa Barbara, CA 93106-4030, USA \\ E-mail: pinnow@theo-phys.uni-essen.de and wiese@itp.ucsb.edu
}

Received 16 October 2001

Published 25 January 2002

Online at stacks.iop.org/JPhysA/35/1195

\begin{abstract}
In this paper we study the effect of a $\delta$-interaction on a polymerized membrane of arbitrary internal dimension $D$. Depending on the dimensionality of the membrane and embedding space, different physical scenarios are observed. We emphasize the difference of polymers from membranes. For the latter, nontrivial contributions appear at the two-loop level. We also exploit a 'massive scheme' inspired by calculations in fixed dimensions for scalar field theories. Despite the fact that these calculations are only amenable numerically, we found that in the limit of $D \rightarrow 2$ each diagram can be evaluated analytically. This property extends in fact to any order in perturbation theory, allowing for a summation of all orders. This is a novel and quite surprising result. Finally, an attempt to go beyond $D=2$ is presented. Applications to the case of selfavoiding membranes are mentioned.
\end{abstract}

PACS numbers: 87.16.Dg, 11.10.-z, 64.60.-i

(Some figures in this article are in colour only in the electronic version)

\section{Introduction}

Interacting lines and more generally manifolds play an important role in many areas of modern physics. Examples of lines are selfavoiding polymers [1], vortex-lines in superconductors [2], directed polymers in a disordered environment, also equivalent to surface growth [3], diffusion of particles and many more. Generalizing results to membranes often poses severe problems, but also new insight into physics. Recently, a lot of work has been devoted to selfavoiding membranes (see [4] for a review). Applications reach as far as high-energy physics, where strings and $M$-branes have been proposed as a general framework for unifying all fundamental interactions.

In this paper, we focus on the statistical physics of systems without disorder. We think of situations like the binding and unbinding of a long chain such as a polymer or a membrane on a wall or the wetting of an interface. More precisely, we study the interaction of a single freely 
fluctuating manifold with another nonfluctuating, fixed object. Depending on whether the interaction is attractive or repulsive, one can distinguish two different scenarios: one may either observe a delocalization transition from an attractive substrate as in wetting phenomena or steric repulsions by a fluctuating manifold. Both cases have in common that excluded volume effects become important. The situation for polymers or a one-dimensional interface is relatively simple: one knows that in this case polymers interacting with a defect or a wall (excluding or not half of the space) as well as short-range critical wetting are in the same universality class [5,6]. For two-dimensional interfaces, the situation is more complicated, and a lot of effort has been spent on understanding for example the delocalization transition [7-14]. Particularly, one is interested in critical wetting for the case of a short-ranged interaction potential. Then, it cannot be approximated by a polynomial in the field $\vec{r}$ and the conventional field-theoretic approach known from $\phi^{4}$-theory fails. This led to a couple of different ansätze [8,9,11-13], among others the functional renormalization group approach of $[12,13]$.

Here we follow a different route: we start by constructing a systematic perturbative renormalization group treatment of the delocalization transition as well as the universal repulsive force exerted by a membrane on a point, line or more generally hyper-plane-like defect. We therefore introduce a flexible 'phantom' manifold of internal dimension $0 \leqslant D \leqslant 2$. By introducing a $\delta$-potential in a subspace $\mathcal{E}$ of dimension $d^{\prime}$, part of the embedding space $\mathbb{R}^{d+d^{\prime}}$ (see figure 1) we punish configurations crossing $\mathcal{E}$. Neglecting the effect of selfavoidance between distinct points within the fluctuating manifold, the free energy of a given configuration reads

$$
\mathcal{H}[\vec{r}]=\int_{x \in \mathcal{M}} \mathrm{d}^{D} x \frac{1}{2}(\nabla \vec{r}(x))^{2}+g_{0} \int_{x \in \mathcal{M}} \mathrm{d}^{D} x \int_{\vec{y} \in \mathcal{E}} \mathrm{d}^{d^{\prime}} \vec{y} \delta^{d+d^{\prime}}(\vec{r}(x)-\vec{y})
$$

where $\vec{r}(x)$ is the position of monomer $x \in \mathcal{M}$ in embedding space $\mathbb{R}^{d+d^{\prime}}$, and $\mathcal{M}$ denotes the $D$-dimensional coordinate space of the manifold. In the case of a polymer this is simply the internal chain length. $g_{0}$ is the attractive or repulsive interaction, of dimension (in inverse length-units)

$$
\varepsilon(D, d):=\left[g_{0}\right]=D-\frac{2-D}{2} d .
$$

The interaction is naively relevant in the infrared for $\varepsilon$ positive, and irrelevant for $\varepsilon$ negative. In a perturbative treatment, $\varepsilon$ is the natural expansion parameter. The situation is similar to selfavoiding membranes and thus (1.1) has been studied as a 'toy model' for the analysis of the renormalizability of the more complicated interaction in the generalized Edwards model for selfavoiding polymerized membranes [15-17]. It was shown to be renormalizable for arbitrary manifold dimensions $0<D<2[16,17]$. This means that the large-scale properties are universal, i.e. depend neither on the regularization scheme used in these calculations, nor on the form of the contact interaction, as long as it is short ranged. Universal quantities have thus been obtained to one-loop order. They are related, as we shall show below, to the correction to scaling exponent $\omega$, which as usual in critical phenomena contains deviations from the long-distance scaling behaviour. Neglecting selfinteractions, one has to distinguish between polymers, which are always crumpled, and membranes, for which a highly folded high-temperature state is separated through a crumpling transition from an essentially flat low-temperature phase [18-21]. The effect of selfavoidance can also be taken into account [4,22-25]. It has been shown to lead as in the case of polymers to a swelling of the membrane and correspondingly to a nontrivial exponent for the radius of gyration [26,27]. However, it is not clear whether a fractal phase can be found in experiments or simulations (see [28] for the latest simulations). In this work we shall mostly neglect selfavoidance. 


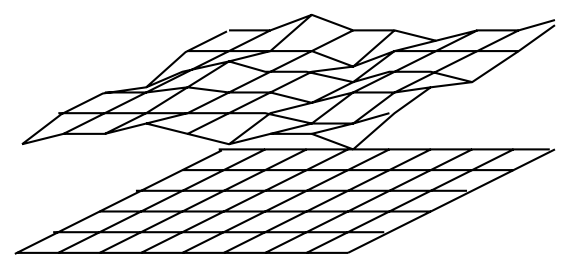

Figure 1. A fluctuating membrane interacting by excluded volume with a surface-like defect.

The aim of this paper is twofold: first, we present the necessary techniques to treat the model (1.1) beyond the leading order. We explicitly perform a two-loop calculation which gives the correction to the scaling exponent $\omega$ at order $\varepsilon^{2}$. Specializing to membranes one finds that the two-loop result naively diverges in the limit of $D \rightarrow 2$. This is a problem of the $\varepsilon$-expansion, since there diagrams have to be evaluated at $\varepsilon\left(D, d_{\mathrm{c}}(D)\right)=0$, and taking $D \rightarrow 2$ implies $d_{\mathrm{c}}(D) \rightarrow \infty$ causing the result to diverge. This motivated us to try a 'massive scheme' in fixed dimension, i.e. finite $\varepsilon$. It turns out that the limit $D \rightarrow 2$ can then be taken and is regular. Even more, the two-loop diagram, which for $D<2$ can only be calculated numerically, can now be evaluated analytically. This striking property even holds for higher orders, and we are able to give an explicit-quite simple-expression for the perturbation series. Then the whole series can be summed and the strong-coupling limit analysed. This is one of the very few cases where one can indeed obtain the exact relation between bare and renormalized coupling in the limit of $D \rightarrow 2$, and thus the exact $\beta$-function. This result does not depend on the explicit regularization and renormalization prescriptions, and is also obtained for a membrane of spherical or toroidal topology. In a final step, we lay the foundations for an expansion about $D=2$. In contrast to the leading order, the first-order corrections already depend on the cutoff procedure. We study one specific procedure, which turns out to reproduce results for polymers at leading order approximately, and even exactly in $d=0$. Work is in progress to obtain a more systematic expansion about $D=2$ [29].

\section{Model and physical observables}

\subsection{The model}

We start from the manifold Hamiltonian (1.1). We split the total embedding space $\mathbb{R}^{d+d^{\prime}}$ into $\mathcal{E}$ and its orthogonal complement $\mathcal{E}_{\perp}$ of dimension $d$. Each $x \in \mathcal{V}$ points to a point $\vec{r}(x)=\left(\vec{r}_{\perp}(x), \vec{r}_{\|}(x)\right)$, with $\vec{r}_{\|}(x) \in \mathcal{E}$ and $\vec{r}_{\perp}(x) \in \mathcal{E}_{\perp}$. The integration over the subspace $\mathcal{E}$ is then trivial and gives

$$
\int_{\vec{y} \in \mathcal{E}} \delta^{d+d^{\prime}}(\vec{r}(x)-\vec{y})=\delta^{d}\left(\vec{r}_{\perp}(x)\right) .
$$

In the partition function

$$
\mathcal{Z}_{\text {total }}=\int \mathcal{D}[\vec{r}] \mathrm{e}^{-\mathcal{H}[\vec{r}]}
$$

the contributions from the parallel and orthogonal components of $\vec{r}(x)$ factorize as

$$
\mathcal{Z}_{\text {total }}=\mathcal{Z}_{0} \times \mathcal{Z}\left(g_{0}\right)
$$

with

$$
\mathcal{Z}_{0}=\int \mathcal{D}\left[\vec{r}_{\|}\right] \mathrm{e}^{-\frac{1}{2}\left(\nabla_{\|} \vec{r}(x)\right)^{2}}
$$




$$
\begin{aligned}
& \mathcal{Z}\left(g_{0}\right)=\int \mathcal{D}\left[\vec{r}_{\perp}\right] \mathrm{e}^{-\mathcal{H}_{\perp}\left[\vec{r}_{\perp}\right]} \\
& \mathcal{H}_{\perp}\left[\vec{r}_{\perp}\right]=\int_{\mathcal{M}} \mathrm{d}^{D} x\left(\frac{1}{2}\left(\nabla \vec{r}_{\perp}(x)\right)^{2}+g_{0} \delta^{d}\left(\vec{r}_{\perp}(x)\right)\right) .
\end{aligned}
$$

Since $\mathcal{Z}_{0}$ is trivial, we shall only consider $\mathcal{H}_{\perp}\left[\vec{r}_{\perp}\right]$ and shall drop the subscript $\perp$ for notational simplicity. We keep in mind that cases with $d<D$ make sense, for instance a polymerized (nonselfavoiding) membrane interacting with a wall is described by (2.6) setting $D=2$ and $d=1$.

Let us discuss (2.6) in more detail: the first term is the elastic energy of the manifold, which is entropic in origin. We have scaled elasticity and temperature to unity. The second term models the interaction of the manifold with a single point at the origin in the external $d$-dimensional space, but we recall that the physical interpretation may well be that of a line or surface. The coupling constant $g_{0}$ may either be positive (repulsive interaction) or negative (attractive interaction). We now give the dimensional analysis. In coordinate-space units, the engineering dimensions are

$$
\begin{aligned}
& \operatorname{dim}[x]=1 \\
& v:=\operatorname{dim}[\vec{r}]=\frac{2-D}{2} \\
& \varepsilon:=\operatorname{dim}\left[\int_{\mathcal{M}} \mathrm{d}^{D} x \delta^{d}(\vec{r}(x))\right]=D-v d \\
& g_{0} \sim \mu^{\varepsilon} \\
& \operatorname{dim}[\mu]=-1
\end{aligned}
$$

where

$$
\mu \equiv \frac{1}{L}
$$

is an inverse length scale. The interaction is naively relevant for $\varepsilon>0$, i.e. $d<d_{\mathrm{c}}$ with (see figure 2)

$$
d_{\mathrm{c}}=\frac{2 D}{2-D}
$$

irrelevant for $\varepsilon<0$ and marginal for $\varepsilon=0$. It has been shown $[16,17]$ that the model is renormalizable for $0<D<2$ and $\varepsilon \geqslant 0$. Results for negative $\varepsilon$ are obtained via analytical continuation. One can define the renormalized coupling $g$ as

$$
g:=\frac{\mathcal{N}}{\mathcal{V}_{\mathcal{M}}}\left[\mathcal{Z}(0)-\mathcal{Z}\left(g_{0}\right)\right] L^{\varepsilon}
$$

where the normalization $\mathcal{N}$ depends on the definition of the path-integral (but not on $L$ ) and is chosen such that

$$
g=g_{0} L^{\varepsilon}+\mathrm{O}\left(g_{0}^{2}\right) .
$$

Universal quantities are obtained at fixed points of the $\beta$-function, which is defined as

$$
\beta(g):=\left.\mu \frac{\partial g}{\partial \mu}\right|_{g_{0}} .
$$

The $\beta$-function thus describes, how the effective coupling $g$ changes under scale transformations, while keeping the bare coupling $g_{0}$ fixed. Let us already anticipate the oneloop result, which we derive later. It reads

$$
\beta(g)=-\varepsilon g+\frac{1}{2} g^{2}+\mathrm{O}\left(g^{3}\right)
$$




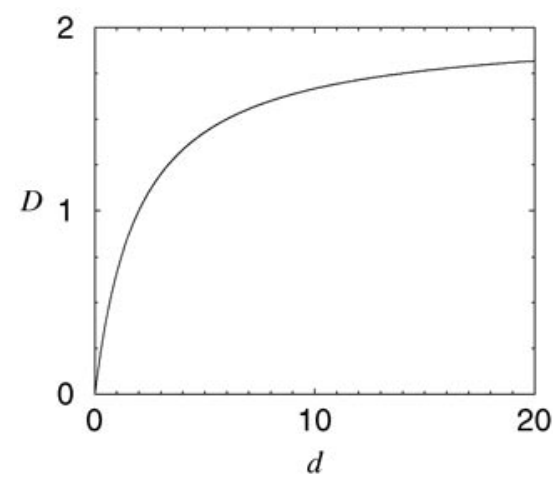

Figure 2. Critical curve defined through $\varepsilon=0 \Leftrightarrow d_{\mathrm{c}}(D)=2 D / 2-D$. The interaction is relevant for points that lie above that curve.

where $g$ is the dimensionless renormalized coupling. Apart from the trivial solution, $g=0$, the flow equation given by (2.12) and (2.13) has a nontrivial fixed point at the zero of the $\beta$-function

$$
g^{*}=2 \varepsilon+\mathrm{O}\left(\varepsilon^{2}\right) .
$$

We shall show below that the scaling behaviour is described by the slope of the RG-function at the fixed point, which is universal as a consequence of renormalizabilty. The long-distance behaviour is then governed by the $\delta$-interaction as considered in our model (2.6), which is the most relevant operator at large scales. Let us now discuss possible physical situations (see figure 3):

(a) $\varepsilon>0$. The RG-flow has an infrared stable fixed point at $g^{*}>0$ and an IR-unstable fixed point at $g=0$. The latter describes an unbinding transition whose critical properties are given by the noninteracting system, while the nontrivial IR stable fixed point determines the long-distance properties of the delocalized state, the long-range repulsive force exerted by the fluctuating manifold on the origin - which we recall may be a point, a line or a plane.

(b) $\varepsilon<0$. Now, the long-distance behaviour is Gaussian, while the unbinding transition occurs at some finite value of the attractive potential, $g^{*}<0$, which corresponds to an infrared unstable fixed point of the $\beta$-function. Below $g^{*}$ the manifold stays always attracted.

(c) $\varepsilon=0$. This is the marginal situation, where the transition takes place at $g^{*}=0$; we expect logarithmic corrections to scaling.

Note that in the presence of an impenetrable wall constraining the configurational space strictly to half of the embedding space, the above considerations should still apply, when shifting the interaction strength appropriately. We shall discuss that in section 2.3.

\subsection{Repulsive force exerted by a membrane on a wall}

Since we are mainly interested in the long-distance properties of membranes for which always $\varepsilon>0$ (this is $(a)$ in figure 3 ), let us try to calculate the repulsive force exerted by the membrane on the origin in the case where this point is strictly forbidden. We shall derive a universal expression for this force [17]. We need the (not normalized) membrane density at position $\vec{r}$

$$
\mathcal{Z}\left(\vec{r}, g_{0}\right):=\frac{1}{\mathcal{V}_{\mathcal{M}}} \int \mathcal{D}[\vec{r}] \int_{\mathcal{M}} \mathrm{d}^{D} x \delta^{d}(\vec{r}(x)-\vec{r}) \mathrm{e}^{-\mathcal{H}} .
$$




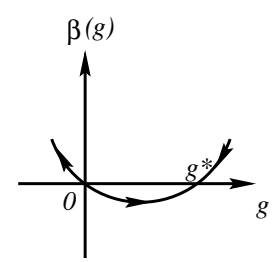

(a)

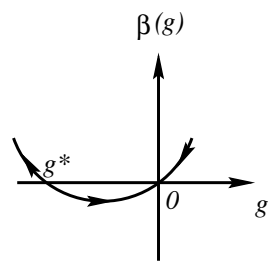

(b)

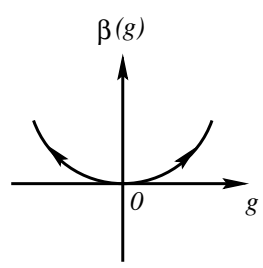

(c)

Figure 3. RG-function and flow for increasing manifold size $L$ for the dimensionless renormalized coupling $g$ : (a) in the case $\varepsilon>0,(b)$ in the case $\varepsilon<0,(c)$ in the case $\varepsilon=0$.

Since the $\delta$-interaction also appears in $\mathcal{H}$, we can relate the density at the origin to the derivative of the partition function with respect to $g_{0}$ :

$$
\mathcal{Z}\left(\overrightarrow{0}, g_{0}\right)=-\left.\frac{1}{\mathcal{V}_{\mathcal{M}}} \frac{\partial}{\partial g_{0}}\right|_{L} \mathcal{Z}\left(g_{0}\right)=\left.\frac{1}{\mathcal{N}} \frac{\partial\left(g L^{-\varepsilon}\right)}{\partial g_{0}}\right|_{L}
$$

where $g$ is the renormalized or effective coupling defined in (2.10). Since $\mathcal{Z}\left(\overrightarrow{0}, g_{0}\right)$ is dimensionless, it depends on the dimensionless combination $g_{0} L^{\varepsilon}$ (and $\varepsilon$ ) only. Using $\mu=1 / L$, we obtain

$$
\mathcal{N} \mathcal{Z}\left(\overrightarrow{0}, g_{0}\right)=\left.L^{-\varepsilon} \frac{\partial g}{\partial g_{0}}\right|_{L} \equiv-\frac{\beta(g)}{\varepsilon g_{0} L^{\varepsilon}} .
$$

For the further considerations, it is sufficient to know the behaviour of the $\beta$-function close to the nontrivial zero. Expanding about $g=g^{*}$, we have

$$
\beta(g)=\left(g-g^{*}\right) \omega\left(g^{*}\right)+\mathrm{O}\left(\left(g-g^{*}\right)^{2}\right) .
$$

Combining (2.16)-(2.18), we get

$$
\left.\frac{\partial g}{\partial\left(g_{0} L^{\varepsilon}\right)}\right|_{L}=-\frac{\beta(g)}{\varepsilon g_{0} L^{\varepsilon}}=-\frac{\left(g-g^{*}\right) \omega\left(g^{*}\right)}{\varepsilon g_{0} L^{\varepsilon}} .
$$

The solution of this differential equation is

$$
g-g^{*} \sim\left(g_{0} L^{\varepsilon}\right)^{-\omega\left(g^{*}\right) / \varepsilon}+\cdots .
$$

Using (2.17), this implies the scaling law

$$
\mathcal{Z}\left(\overrightarrow{0}, g_{0}\right) \sim\left(g_{0}^{1 / \varepsilon} L\right)^{-\omega\left(g^{*}\right)-\varepsilon} .
$$

We now use this result to derive a scaling law for $\mathcal{Z}\left(\vec{r}, g_{0}\right)$, with $\vec{r} \neq \overrightarrow{0}$. In addition to $g_{0} L^{\varepsilon}$ (and $\varepsilon), \mathcal{Z}\left(\vec{r}, g_{0}\right)$ depends on $\vec{r}$; taking into account rotational symmetry and dimensionality, it depends on $\vec{r}$ only through the dimensionless combination $r / L^{v}$, with $r=|\vec{r}|$ :

$$
\mathcal{Z}\left(\vec{r}, g_{0}\right)=\mathcal{Z}\left(r / L^{\nu}, g_{0} L^{\varepsilon}\right) .
$$

For $\mathcal{Z}\left(\vec{r}, g_{0}\right)$, the most interesting limit is that of $g_{0} \rightarrow \infty$. Physically, this corresponds to strictly forbidding monomers to be at the origin. Therefore it is clear that this limit is well behaved, and

$$
\mathcal{Z}_{\infty}\left(r / L^{\nu}\right):=\lim _{g_{0} \rightarrow \infty} \mathcal{Z}\left(r / L^{\nu}, g_{0} L^{\varepsilon}\right)
$$

is finite. In order to be consistent with (2.21) it has to obey a power law in the scaling regime $r \ll L^{v}$

$$
\mathcal{Z}_{\infty}\left(r / L^{v}\right) \sim\left(r / L^{\nu}\right)^{\theta}
$$


Comparing the $L$-dependence of $\mathcal{Z}_{\infty}\left(r / L^{\nu}\right)$ and $\mathcal{Z}\left(\overrightarrow{0}, g_{0}\right)$, we obtain the exponent identity

$$
\theta=\frac{\varepsilon+\omega\left(g^{*}\right)}{v} \text {. }
$$

Finally, from (2.24) we derive the repulsive force between the origin and the manifold

$$
\vec{f}(\vec{r})=\nabla_{\vec{r}} \ln \mathcal{Z}_{\infty}\left(|\vec{r}| / L^{v}\right)=\theta \frac{\vec{r}}{r^{2}} .
$$

Note that to derive this result, $k_{\mathrm{B}} T$ has been set to 1 . Reestablishing the temperature dependence, we find

$$
\vec{f}(\vec{r})=k_{\mathrm{B}} T \theta \frac{\vec{r}}{r^{2}} .
$$

Also note that this argument gives $\theta=0$ at the Gaussian fixed point, which is necessary since for $g_{0}=0$ no force is exerted on the membrane.

\subsection{Unbinding transition}

Let us discuss the physical situation at the UV-stable fixed point in figure 3. The fixed point corresponds to a delocalization transition of the manifold, which is at vanishing coupling $g^{*}=0$ for $\varepsilon>0$ and at some finite attractive coupling $g^{*}<0$ for $\varepsilon<0$.

In the localized phase $g<g^{*}$, correlation functions such as $\left\langle[\vec{r}(x)-\vec{r}(y)]^{2}\right\rangle$ and the associated correlation length $\xi_{\|}$(in the $D$-dimensional internal space) should be finite, as well as the radius of gyration $\xi_{\perp}$. Approaching the transition point these quantities diverge as [14]

$$
\xi_{\|} \sim\left(g^{*}-g\right)^{-v_{\|}} \quad \xi_{\perp} \sim\left(g^{*}-g\right)^{-v_{\perp}} .
$$

Since $\xi_{\perp} \sim \xi_{\|}^{\nu}$, the exponents $\nu_{\|}$and $\nu_{\perp}$ are related through

$$
v_{\perp}=v_{\|} v
$$

$v$ being the dimension of the field (2.7). To relate $v_{\|}$to $\omega$, we first observe that $\xi_{\|}$depends only on $g_{0}$ and the membrane size $L$, or its inverse $\mu=1 / L$. Writing $\xi_{\|}$as a function of the renormalized coupling $g$ and the scale $\mu$, we thus have

$$
\begin{aligned}
0 & =\mu \frac{\partial}{\partial \mu} \xi_{\|}(g, \mu) \\
& =\mu \frac{\partial}{\partial \mu}\left(\frac{1}{\mu} f(g)\right) \\
& =\frac{1}{\mu}\left(-f(g)+\beta(g) f^{\prime}(g)\right)
\end{aligned}
$$

where the dimension-full factor $1 / \mu$ has been factored from the dependence on the dimensionless renormalized coupling $g$. Using that $\frac{\partial}{\partial g} \xi_{\|}(g)=\frac{1}{\mu} f^{\prime}(g)$, this can be rewritten as

$$
\begin{aligned}
\xi_{\|}(g) & =\beta(g) \frac{\partial}{\partial g} \xi_{\|}(g) \\
& \approx \omega\left(g^{*}\right)\left(g-g^{*}\right) \frac{\partial}{\partial g} \xi_{\|}(g) .
\end{aligned}
$$

The solution to the above equation is

$$
\xi_{\|} \sim\left|g-g^{*}\right|^{1 / \omega\left(g^{*}\right)} .
$$

This leads to the identification

$$
v_{\|}=-\frac{1}{\omega\left(g^{*}\right)} \quad v_{\perp}=-\frac{v}{\omega\left(g^{*}\right)} .
$$




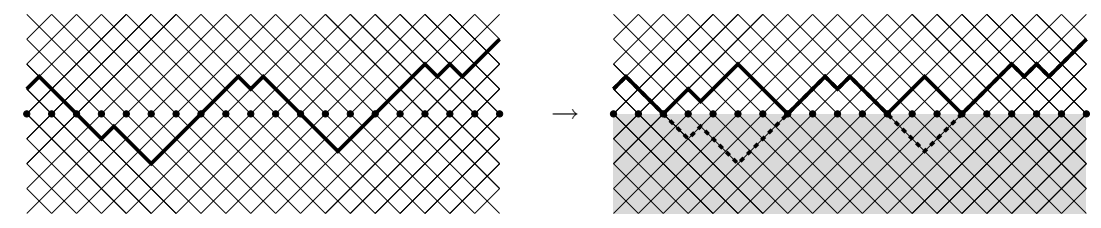

Figure 4. A polymer (here for simplicity directed) in interaction with a wall (thick dots). Left before and right after flapping up. The grey area is impenetrable.

Note that $\omega\left(g^{*}\right)<0$ at the transition. Specializing to $(D, d)=(1,1)$, we find

$$
v_{\perp}=1 \quad v_{\|}=2 .
$$

These exponents are also valid for the delocalization transition of a one-dimensional interface from an attractive hard wall in two-dimensional bulk space $[6,8,14]$. This can be understood as follows: the partition function of a fluctuating polymer interacting with a $\delta$-defect at the boundary of a hard wall can be written as

$$
\mathcal{Z}=\frac{1}{2} \sum_{n=0}^{\infty} \mathcal{Z}_{n}\left(\frac{1}{2}\right)^{n} \mathrm{e}^{-n \beta E}
$$

where the sum runs over the number of contacts with the defect hyper-plane. $E$ denotes the contact energy, $\beta=\left(k_{\mathrm{B}} T\right)^{-1}$ and $\mathcal{Z}_{n}$ is the constrained partition function of the free interface having exactly $n$ points of contact with the defect. The powers of $\frac{1}{2}$ only appear in the presence of an impenetrable defect and reflect the fact that all configurations in the presence of a hard wall can be obtained by flapping up those parts of the polymer which have penetrated the line (see figure 4 ). This accounts for a factor $\frac{1}{2}$ for each possible flap. Thus, the hard-wall constraint translates into a shift in the binding energy according to

$$
E_{\text {no wall }} \longrightarrow E_{\text {wall }}=E_{\text {no wall }}+k_{\mathrm{B}} T \ln 2 \text {. }
$$

The delocalization transition occurring for $(D, d)=(1,1)$ at vanishing potential strength is shifted to an attractive interaction dependent on the temperature. Due to the correspondence (2.36) the exponents characterizing the transition remain unchanged.

\section{Operator product expansion and one-loop result}

The partition function of the model has been defined in (2.5). Analogously, we define the expectation value of an observable $\mathcal{O}(r)$ as (we denote $\vec{r}$ by $r$ from now on)

$$
\langle\mathcal{O}(r)\rangle_{g_{0}}:=\frac{\int \mathcal{D}[r] \mathrm{e}^{-\mathcal{H}[r]}}{Z\left(g_{0}\right)} .
$$

Most physical observables can be derived from expectation values of general $M$-point vertex operators like

$$
\mathcal{O}(r)=\prod_{j=1}^{M} \mathrm{e}^{\mathrm{i} p_{j} r\left(s_{j}\right)} .
$$

Before we go on, let us make some changes in normalizations, which will be helpful in the following. First, we rescale the field and the coupling constant according to

$$
r \longrightarrow(2-D)^{1 / 2} r \quad g_{0} \longrightarrow(2-D)^{d / 2} g_{0} .
$$


Second, we change the integration over internal coordinates to

$$
\int_{x}:=\frac{1}{S_{D}} \int \mathrm{d}^{D} x \quad S_{D}=2 \frac{\pi^{D / 2}}{\Gamma(D / 2)}
$$

being the volume of the $D$-dimensional unit-sphere. Third, the normalization of the $\delta$ distribution is changed to

$$
\tilde{\delta}^{d}(r(x)):=(4 \pi)^{d / 2} \delta(r(x))=\int_{k} \mathrm{e}^{\mathrm{i} k r(x)}
$$

with

$$
\int_{k}:=\pi^{-d / 2} \int \mathrm{d}^{d} k
$$

The advantage of these normalizations is that

$$
\int_{k} \mathrm{e}^{-k^{2}}=1 \quad \int_{x} \Theta(|x|<1)=1 .
$$

The model in the new normalizations now reads

$$
\mathcal{H}[r]=\frac{1}{2-D} \int_{x} \frac{1}{2}(\nabla r(x))^{2}+g_{0} \int_{x} \tilde{\delta}^{d}(r(x))
$$

and (due to the factor of $1 /(2-D)$ in the above definition) the two-point correlator is

$$
C\left(x_{i}-x_{j}\right):=\frac{1}{2 d}\left\langle\left[r\left(x_{i}\right)-r\left(x_{j}\right)\right]^{2}\right\rangle_{0}=\left|x_{i}-x_{j}\right|^{2 v}
$$

We now proceed with the calculation of physical observables. As an explicit example, let us consider the perturbation expansion of the one-point vertex operator

$$
\mathcal{Z}^{(p)}:=\mathcal{Z}\left(g_{0}\right)\left\langle\mathrm{e}^{\mathrm{i} p r(s)}\right\rangle_{g_{0}}=\sum_{N=0}^{\infty} \frac{\left(-g_{0}\right)^{N}}{N !} \mathcal{Z}_{N}^{(p)}
$$

where

$$
\mathcal{Z}_{N}^{(p)}=\left\langle\mathrm{e}^{\mathrm{i} p r(s)} \prod_{i=1}^{N} \int_{x_{i}} \tilde{\delta}^{d}\left(r\left(x_{i}\right)\right)\right\rangle_{0} .
$$

This can be written as

$$
\mathcal{Z}_{N}^{(p)}=\prod_{i=1}^{N} \int_{x_{i}} \int_{k_{i}} \tilde{\delta}^{d}\left(p+\sum k_{i}\right)\left\langle\mathrm{e}^{\mathrm{i}\left(\sum_{i=1}^{N} k_{i} r\left(x_{i}\right)+\operatorname{pr}(s)\right)}\right\rangle_{0}
$$

where we have already integrated out a global translation of the field $r$. The Gaussian average is

$$
\left\langle\mathrm{e}^{\mathrm{i}\left(\sum_{i=1}^{N} k_{i} r\left(x_{i}\right)+\operatorname{pr}(s)\right)}\right\rangle_{0}=\mathrm{e}^{\frac{1}{2} \sum_{i, j=0}^{N} k_{i} k_{j} C\left(x_{i}-x_{j}\right)} \quad k_{0}=p .
$$

The $\mathcal{Z}_{N}^{(p)}$ in (3.11) possess short-distance singularities for $N \geqslant 2$. In order to analyse these singularities we shall use the techniques of normal ordering and operator product expansion in the following. In our problem the procedure of normal ordering a vertex operator $\mathrm{e}^{\mathrm{i} k_{j} r\left(x_{j}\right)}$ turns out to be quite simple: in any expectation value, we factorize out the contractions between the vertex operators. At one-loop order or equivalently at second order in (3.10) this means that

$$
\mathrm{e}^{\mathrm{i} k_{1} r\left(x_{1}\right)} \mathrm{e}^{\mathrm{i} k_{2} r\left(x_{2}\right)}=: \mathrm{e}^{\mathrm{i}\left(k_{1} r\left(x_{1}\right)+k_{2} r\left(x_{2}\right)\right)}: \mathrm{e}^{k_{1} k_{2} C\left(x_{1}-x_{2}\right)} .
$$

This can be understood as a definition of the normal-ordered product $: \mathrm{e}^{\mathrm{i}\left(k_{1} r\left(x_{1}\right)+k_{2} r\left(x_{2}\right)\right)}:$. Note that equality in the above equation is meant in the sense of operators, that is when inserted into expectation values within the free theory. 
Let us now turn to an explicit derivation of the OPE of two $\delta$-interactions, i.e. we study (3.14) for small distances $x_{1}-x_{2}$. Since the short-distance singularities appear only in the internal contractions, the normal-ordered product in the rhs of (3.14) is regular for small distances $x_{1}-x_{2}$ and thus can be expanded therein. We then project the resulting terms on the corresponding operators. For that purpose we make a change in the momentum variables according to

$$
\left(\begin{array}{l}
k_{1} \\
k_{2}
\end{array}\right) \longrightarrow\left(\begin{array}{c}
\frac{k_{1}}{2}-k_{2} \\
\frac{k_{1}}{2}+k_{2}
\end{array}\right)
$$

In internal space we change coordinates to the centre of mass system:

$$
\bar{x}:=\frac{1}{2}\left(x_{1}+x_{2}\right) \quad y:=x_{2}-x_{1} .
$$

Thus, the rhs of (3.14) takes the form

$$
\begin{aligned}
: \mathrm{e}^{\mathrm{i}\left[\left(k_{1} / 2-k_{2}\right) r(\bar{x}-y / 2)+\left(k_{1} / 2+k_{2}\right) r(\bar{x}+y / 2)\right]}: \mathrm{e}^{\left(k_{1}^{2} / 4-k_{2}^{2}\right) C(y)} & : \mathrm{e}^{\mathrm{i} k_{1} r(\bar{x})}\left(1+\mathrm{i} k_{2} y \nabla r(\bar{x})+\mathrm{i} k_{1} \frac{1}{2 !} \frac{y^{\alpha} y^{\beta}}{4} \nabla_{\alpha} \nabla_{\beta} r(\bar{x})-\frac{\left(k_{2} y \nabla r(\bar{x})\right)^{2}}{2 !}+\cdots\right) \\
& \times\left(1+\frac{k_{1}^{2}}{4} C(y)+\cdots\right) \mathrm{e}^{-k_{2}^{2} C(y)}
\end{aligned}
$$

Integration over $k_{1}, k_{2}$ yields the OPE of $\tilde{\delta}^{d}\left(r\left(x_{1}\right)\right)$ with $\tilde{\delta}^{d}\left(r\left(x_{2}\right)\right)$ :

$$
\begin{aligned}
\tilde{\delta}^{d}\left(r\left(\bar{x}-\frac{y}{2}\right)\right) & \tilde{\delta}^{d}\left(r\left(\bar{x}+\frac{y}{2}\right)\right)=y^{-v d} \tilde{\delta}^{d}(r(\bar{x}))-y^{-v d+2 v} \frac{1}{4} \partial_{r}^{2} \tilde{\delta}^{d}(r(\bar{x})) \\
& -y^{-v d-2 v+2} \frac{1}{4 D} \tilde{\delta}^{d}(r(\bar{x}))(\nabla r(\bar{x}))^{2} \\
& -y^{-v d+2} \frac{1}{16 D}\left(-\Delta_{r}\right) \tilde{\delta}^{d}(r(\bar{x}))(\nabla r(\bar{x}))^{2} \\
& +y^{-v d+2} \frac{1}{8 D} \partial_{r_{\mathrm{i}}} \tilde{\delta}^{d}(r(\bar{x})) \Delta r_{\mathrm{i}}(\bar{x})+\cdots
\end{aligned}
$$

Restricting ourselves to the most relevant next to leading operators for internal dimension $D$ smaller than, but close to 2 , the operator product expansion reads

$\tilde{\delta}^{d}\left(r\left(\bar{x}-\frac{y}{2}\right)\right) \tilde{\delta}^{d}\left(r\left(\bar{x}+\frac{y}{2}\right)\right)=y^{-v d} \tilde{\delta}^{d}(r(\bar{x}))+\sum_{n=1}^{\infty} y^{-v d+n(D-2)} \frac{\left(-\partial_{r}^{2}\right)^{n} \tilde{\delta}^{d}(r(\bar{x}))}{4^{n} n !}$.

The OPE-coefficient in front of the leading operator, which is the $\delta$-interaction itself, carries the leading short-distance singularity. In the following we introduce some diagrammatic notation. Denoting $\bullet:=\tilde{\delta}^{d}(r(x))$ we abbreviate the projection of two nearby $\delta$-interactions on the $\delta$-interaction itself as

$$
(\bullet \bullet \bullet)=y^{-v d} \text {. }
$$

We now analyse short-distance divergences of the perturbation expansion using the OPE in (3.18). Being interested in the leading scaling behaviour, we drop all subdominant terms for $0<D<2$, such that we only need the leading OPE-coefficient (3.20). The integral over the relative distance in $\int_{y}(\bullet \bullet \bullet) \bullet$ is logarithmically divergent for $\varepsilon=0$. In order to define a counter-term we use dimensional regularization, i.e. we set $\varepsilon>0$. The integral is then UV convergent, but IR divergent. An IR regulator or equivalently a subtraction scale $\mu=L^{-1}$ has to be introduced to define the subtraction operation. Generally, we integrate over all distances 
bound by the inverse subtraction scale $L$. The OPE-coefficient in (3.20) then reduces to a number as

$$
\left(\bullet \bullet|\bullet|_{L}:=\int_{|y|<L}(\bullet \bullet \mid \bullet)=L^{\varepsilon} f(\varepsilon, D) .\right.
$$

Let us use the scheme of minimal subtraction (MS). The internal dimension of the membrane is fixed and (3.21) is expanded in a Laurent series in $\varepsilon$, starting here at order $\varepsilon^{-1}$. Denoting the term of order $\varepsilon^{p}$ in $\left.\langle\mid\rangle_{L}\right|_{L=1}$ by $\langle\mid\rangle_{\varepsilon^{p}}$, the simple pole in (3.21) is

$$
(\bullet \bullet \bullet)_{\varepsilon^{-1}}=\frac{1}{\varepsilon} \text {. }
$$

Of course, in this case, due to our normalizations, this is exact. Let us rewrite the Hamiltonian in (2.6) in terms of the renormalized dimensionless coupling $g$. For perturbative calculations it is customary to write

$$
g_{0}=g Z_{g} \mu^{\varepsilon}
$$

where $Z_{g}$ is the renormalization factor, which is fixed by demanding that observables remain finite in the limit of $\varepsilon \rightarrow 0$. The Hamiltonian becomes

$$
\mathcal{H}[r]=\frac{1}{2-D} \int_{x} \frac{1}{2}(\nabla r(x))^{2}+\int_{x} g Z_{g} \mu^{\varepsilon} \tilde{\delta}^{d}(r(x)) .
$$

We find to one-loop order

$$
Z_{g}=1+\frac{g}{2}\langle\bullet \bullet \bullet\rangle_{\varepsilon^{-1}}+\mathrm{O}\left(g^{2}\right)
$$

There is no field-renormalization since the elastic part has also to describe the manifold far away from the origin, for which the interaction term can be neglected. Formally, of course, this follows from the OPE. The renormalization group $\beta$-function is defined as

$$
\beta(g):=\left.\mu \frac{\mathrm{d}}{\mathrm{d} \mu}\right|_{g_{0}} g
$$

from which it follows together with (3.23) that

$$
\beta(g)=\frac{-\varepsilon g}{1+g \frac{\partial}{\partial g} \ln Z_{g}} .
$$

To one-loop order we obtain from (3.27) and (3.25) as anticipated earlier

$$
\beta(g)=-\varepsilon g+\frac{g^{2}}{2}+\mathrm{O}\left(g^{3}\right) .
$$

The long-distance behaviour of the theory is governed by the IR-stable fixed point of the RG-flow. Fixed points are zeros of the $\beta$-function:

$$
\beta\left(g^{*}\right)=0 \quad \Rightarrow \quad g^{*}=0 \text { or } 2 \varepsilon .
$$

In section 2.2, we defined the correction-to-scaling exponent $\omega$. It is obtained from the slopefunction $\omega(g)$, defined as

$$
\omega(g):=\frac{\mathrm{d}}{\mathrm{d} g} \beta(g)
$$

The correction to the scaling exponent is $\omega(g)$ evaluated at the fixed points, and is found to be

$$
\omega\left(g^{*}=0\right)=-\varepsilon \quad \omega\left(g^{*}=2 \varepsilon\right)=\varepsilon .
$$

Of course, this result is apart from the very existence of a fixed point rather trivial, since it is determined through the leading term of the $\beta$-function, which is always $-\varepsilon g$. In order to obtain any nontrivial information from the perturbation series it is thus necessary to go beyond the leading order. 


\section{Two-loop calculation in a MS-scheme}

\subsection{Operator product expansion}

Let us now continue with the calculation at the two-loop order. At each order of perturbation theory there is only one new diagram. At two-loop order this comes from three coalescing $\delta$-interactions. Again, let us rewrite the product of the three vertex operators as its expectation value times the normal ordered product:

$\mathrm{e}^{\mathrm{i} k_{1} r\left(x_{1}\right)} \mathrm{e}^{\mathrm{i} k_{2} r\left(x_{2}\right)} \mathrm{e}^{\mathrm{i} k_{3} r\left(x_{3}\right)}=: \mathrm{e}^{\mathrm{i}\left[k_{1} r\left(x_{1}\right)+k_{2} r\left(x_{2}\right)+k_{3} r\left(x_{3}\right)\right]}: \mathrm{e}^{k_{1} k_{2} C\left(x_{1}-x_{2}\right)} \mathrm{e}^{k_{1} k_{3} C\left(x_{1}-x_{3}\right)} \mathrm{e}^{k_{2} k_{3} C\left(x_{2}-x_{3}\right)}$.

We use the following change of coordinates:

$$
\left.\begin{array}{rl}
\bar{x} & :=\frac{1}{3}\left(x_{1}+x_{2}+x_{3}\right) \\
a & :=x_{2}-x_{1} \\
b & :=x_{3}-x_{2} \\
c & :=x_{1}-x_{3}
\end{array}\right\} \quad \Leftrightarrow \quad\left\{\begin{array}{l}
x_{1}=\bar{x}+\frac{1}{3}(c-a) \\
x_{2}=\bar{x}+\frac{1}{3}(a-b) \\
x_{3}=\bar{x}+\frac{1}{3}(b-c) .
\end{array}\right.
$$

As at one-loop order, : $\mathrm{e}^{\mathrm{i}\left[k_{1} r\left(x_{1}\right)+k_{2} r\left(x_{2}\right)+k_{3} r\left(x_{3}\right)\right]}:$ is free of divergences upon approaching the points $x_{i}$, whereas the UV-divergence comes from the factor $\mathrm{e}^{k_{1} k_{2} C\left(x_{1}-x_{2}\right)} \mathrm{e}^{k_{1} k_{3} C\left(x_{1}-x_{3}\right)} \mathrm{e}^{k_{2} k_{3} C\left(x_{2}-x_{3}\right)}$. The leading term is thus obtained upon setting $x_{1}=x_{2}=x_{3}=\bar{x}$ in : $\mathrm{e}^{\mathrm{i}\left[k_{1} r\left(x_{1}\right)+k_{2} r\left(x_{2}\right)+k_{3} r\left(x_{3}\right)\right]}:$, dropping the gradient terms. Further shifting $k_{1} \rightarrow k_{1}-k_{2}-k_{3}$, we obtain

$$
\mathrm{e}^{\mathrm{i} k_{1} r(\bar{x})} \mathrm{e}^{\left(k_{1}-k_{2}-k_{3}\right) k_{2} C(a)+k_{2} k_{3} C(b)+\left(k_{1}-k_{2}-k_{3}\right) k_{3} C(c)} .
$$

Integrating over $k_{1}, k_{2}$ and $k_{3}$ yields the final result

$\tilde{\delta}^{d}\left(r\left(x_{1}\right)\right) \tilde{\delta}^{d}\left(r\left(x_{2}\right)\right) \tilde{\delta}^{d}\left(r\left(x_{3}\right)\right)=(\bullet \bullet \bullet) \tilde{\delta}^{d}(r(\bar{x}))+\left(\bullet \bullet \bullet \bullet^{\prime \prime}\right)\left(-\partial_{r}^{2}\right) \tilde{\delta}^{d}(r(\bar{x}))+\cdots$

where the OPE-coefficients are given by

$$
\begin{aligned}
(\bullet \bullet) & =\left[C(a) C(c)-\frac{1}{4}(C(a)+C(c)-C(b))^{2}\right]^{-d / 2} \\
& =\left[\frac{1}{4}\left(a^{v}+b^{v}+c^{v}\right)\left(b^{v}+c^{v}-a^{v}\right)\left(a^{v}+c^{\nu}-b^{\nu}\right)\left(a^{v}+b^{v}-c^{\nu}\right)\right]^{-d / 2}
\end{aligned}
$$

which contributes to the renormalization factor at two-loop order. The first subleading term is (denoting $\left.\bullet^{\prime \prime}:=\left(-\partial_{r}^{2}\right) \tilde{\delta}^{d}(r(\bar{x}))\right)$

$$
\begin{aligned}
\left(\bullet \bullet \bullet^{\prime \prime}\right) & =-\frac{1}{4} C(a) C(b) C(c) \\
& \times\left[\frac{1}{4}\left(a^{v}+b^{v}+c^{v}\right)\left(b^{v}+c^{v}-a^{v}\right)\left(a^{v}+c^{v}-b^{v}\right)\left(a^{v}+b^{v}-c^{v}\right)\right]^{-d / 2-1} .
\end{aligned}
$$

\subsection{Numerical calculation in $0<D<2$}

Let us now turn to the explicit calculation of the second-order contribution to the coupling constant renormalization. To two-loop order the renormalization group $Z_{g}$-factor for the coupling constant reads

$$
\begin{aligned}
Z_{g}=1+\frac{g}{2}\left\langle\bullet \bullet|\bullet|_{\varepsilon^{-1}}-\frac{g^{2}}{6}\left[\left(\bullet \bullet|\bullet|_{\varepsilon^{-2}, \varepsilon^{-1}}-\frac{3}{2}\left\langle\bullet \bullet|\bullet|_{\varepsilon^{-1}}^{2}\right]\right.\right.\right. \\
+\frac{g^{2}}{4}\left\langle\bullet \bullet|\bullet|_{\varepsilon^{-1}}^{2}+\mathrm{O}\left(g^{3}\right) .\right.
\end{aligned}
$$


Note that this $Z_{g}$-factor can either be obtained from (2.10) and (3.23) or by expanding the perturbative series and checking that divergent contributions proportional to $\tilde{\delta}(r(x))$ cancel.

The formula is arranged such that in the brackets the second-order pole in $\varepsilon$ cancels. This is because, to leading order in $1 / \varepsilon$, the two-loop diagram factorizes into two one-loop diagrams. The combinatorial factor of $3 / 2$ is composed of a factor of 3 for the number of possible subdivergences, and a factor of $1 / 2$ for the nested integration: the subdivergence in the distance say $a$ only appears in the sector (i.e. part of the domain of integration) in which $a$ is smallest.

Let us define

$$
I_{2}(D, L):=\langle\bullet \bullet \bullet\rangle_{L}-\frac{3}{2}\langle\bullet \bullet \mid \bullet\rangle_{L}^{2}
$$

Let us already note that we shall later calculate in a 'massive' scheme in fixed dimension $d$. In order to use the results derived here, we keep $d$ arbitrary instead of setting $d=d_{\mathrm{c}}$. We now want to derive an expression which can be integrated numerically. Since the bounds on the integrations in (4.8) are asymmetric (recall that in $c$ are bounded by $L$, whereas $\left\langle\bullet|\bullet|_{L}^{2}\right.$ is an integral with two bounds only) we treat both terms separately. We start with the first one, which can be written as

$$
I_{2}^{M}(D, L)=\int_{a, b, c} \Theta(a \leqslant L) \Theta(b \leqslant L) \Theta(c \leqslant L)(\bullet \bullet \bullet) .
$$

The divergence when integrating over the global scale is eliminated by noting that $L \frac{\partial}{\partial L} I_{2}(D, L)=2 \varepsilon I_{2}(D, L)$. There remain of course the sub-divergences, which will be subtracted later by the counter-term. Applying this procedure to (4.9), we obtain

$$
\begin{aligned}
2 \varepsilon I_{2}^{M}(D, L)= & \int_{a, b, c}[\delta(a-L) \Theta(b \leqslant L) \Theta(c \leqslant L)+\delta(c-L) \Theta(b \leqslant L) \Theta(a \leqslant L) \\
& +\delta(b-L) \Theta(a \leqslant L) \Theta(c \leqslant L)](\bullet \bullet) .
\end{aligned}
$$

Since the integral is symmetric in $a, b$ and $c$, we can replace it by three times the first term. Introducing proper normalizations, an explicit representation for the measure and factoring out the explicit $L$-dependence, we obtain

$$
2 \varepsilon I_{2}^{M}(D, L)=\left.3 L^{2 \varepsilon} \frac{S_{D-1}}{S_{D}} \int_{-\infty}^{\infty} \mathrm{d} c_{\|} \int_{0}^{\infty} \mathrm{d} c_{\perp} c_{\perp}^{D-2} \Theta(b \leqslant 1) \Theta(c \leqslant 1)(\bullet \bullet \bullet)\right|_{a=1, b, c} .
$$

We also have to derive an expression for the counter-term. It can be written as

$$
I_{2}^{C}(D, L)=-\frac{3}{2} \int_{a} \int_{c} a^{-v d} c^{-v d} \Theta(a \leqslant L) \Theta(c \leqslant L) .
$$

Using the same trick to derive w.r.t. the IR-regulator as above, we obtain

$2 \varepsilon I_{2}^{C}(D, L)=-\frac{3}{2} L^{2 \varepsilon} \int_{\mathcal{A}, \mathcal{B}, \mathcal{C}} a^{-v d} c^{-v d}[\delta(a-1) \Theta(c \leqslant a)+\delta(c-1) \Theta(a \leqslant c)]$.

Using the technique of conformal mapping, which is presented in appendix $\mathrm{B}$, both integrals above can be transformed into one having support in the single sector $\mathcal{A}$ defined through $b, c \leqslant a$ (where here $a$ is set to 1 ). Analogously, we define sectors $\mathcal{B}$ and $\mathcal{C}$ as the subset of domains where $b$ respectively $c$ is the longest distance. Note that the first term 


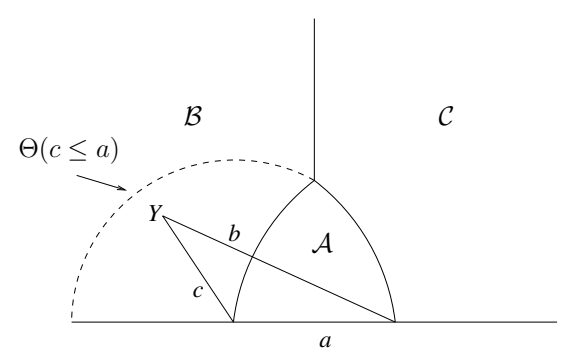

Figure 5. Area of integration (dashed) of the first integral in (4.13). The intersection with $\mathcal{B}$ is to be mapped onto $\mathcal{A}$.

in (4.13) only has contributions from $\mathcal{A}$ and $\mathcal{B}$ (figure 5), while the second can be restricted to $\mathcal{A}$ and $\mathcal{C}$ after renaming $a$ as $b$ and $c$ as $a$.

Performing explicitly the mapping of both $\mathcal{B}$ and $\mathcal{C}$ onto $\mathcal{A}$ one obtains

$$
\begin{aligned}
& \left.\int_{\mathcal{B}}\left(a^{-v d} c^{-v d} \Theta(c \leqslant a)\right)\right|_{a=1}=\int_{\mathcal{A}} b^{-v d-2 \varepsilon} c^{-v d} \Theta(c \leqslant b) \\
& \int_{\mathcal{C}}\left(a^{-v d} b^{-v d} \Theta(b \leqslant a)\right)_{\mid a=1}=\int_{\mathcal{A}} c^{-v d-2 \varepsilon} b^{-v d} \Theta(b \leqslant c) .
\end{aligned}
$$

Combining the contributions from all sectors, we arrive at

$$
2 \varepsilon I_{2}^{C}(D, L)=-\frac{3}{2} L^{2 \varepsilon} \int_{\mathcal{A}}\left(b^{-v d}+c^{-v d}+b^{-v d} c^{-v d} \max (b, c)^{-2 \varepsilon}\right) .
$$

Combining (4.11) and (4.15), we obtain an expression for the complete diagram

$$
\begin{aligned}
2 \varepsilon I_{2}(D, L)= & 3 L^{2 \varepsilon} \frac{S_{D-1}}{S_{D}} \int_{-\infty}^{\infty} \mathrm{d} c_{\|} \int_{0}^{\infty} \mathrm{d} c_{\perp} c_{\perp}^{D-2} \Theta(b \leqslant 1) \Theta(c \leqslant 1) \\
& \times\left\{\left[\frac{1}{4}\left(1+b^{\nu}+c^{\nu}\right)\left(b^{\nu}+c^{\nu}-1\right)\left(1+c^{\nu}-b^{\nu}\right)\left(1+b^{\nu}-c^{\nu}\right)\right]^{-d / 2}\right. \\
& \left.-\frac{1}{2}\left[b^{-v d}+c^{-\nu d}+b^{-v d} c^{-\nu d} \max (b, c)^{-2 \varepsilon}\right]\right\} .
\end{aligned}
$$

This expression will also be used later in a massive scheme. Since here we are only interested in the $\varepsilon$-expansion, we take the limit of $\varepsilon \rightarrow 0$ in the integrand. We have made the (nontrivial) check that the integrand remains a finite integrable function, which can be integrated numerically:

$$
\begin{aligned}
I_{2}(D, L)=3 & \frac{L^{2 \varepsilon}}{2 \varepsilon} \frac{S_{D-1}}{S_{D}} \int_{-\infty}^{\infty} \mathrm{d} c_{\|} \int_{0}^{\infty} \mathrm{d} c_{\perp} c_{\perp}^{D-2} \Theta(b \leqslant 1) \Theta(c \leqslant 1) \\
& \times\left\{\left[\frac{1}{4}\left(1+b^{\nu}+c^{\nu}\right)\left(b^{\nu}+c^{\nu}-1\right)\left(1+c^{\nu}-b^{\nu}\right)\left(1+b^{\nu}-c^{\nu}\right)\right]^{-d_{\mathrm{c}} / 2}\right. \\
& \left.-\frac{1}{2}\left[b^{-v d_{\mathrm{c}}}+c^{-v d_{\mathrm{c}}}+b^{-\nu d_{\mathrm{c}}} c^{-\nu d_{\mathrm{c}}}\right]\right\}+\mathrm{O}\left(\varepsilon^{0}\right) .
\end{aligned}
$$

Let us now proceed with the explicit numeric evaluation of (4.17). There are integrable singularities both in $c_{\|}$and $c_{\perp}$. To disentangle them [27,30] we make a change of variables from Cartesian coordinates $\left(c_{\|}, c_{\perp}\right)$ to radial and angular coordinates $(c, \alpha)$ according to

$$
c_{\|}=c \cos (\alpha) \quad c_{\perp}=c \sin (\alpha)
$$

such that

$$
\int_{-\infty}^{\infty} \mathrm{d} c_{\|} \int_{0}^{\infty} \mathrm{d} c_{\perp} c_{\perp}^{D-2} \longrightarrow \int_{0}^{1} \mathrm{~d} c c^{D-1} \int_{0}^{\pi / 2} \mathrm{~d} \alpha(\sin (\alpha))^{D-2}
$$

where the upper bounds of $\pi / 2$ on $\alpha$ and 1 on $c$ are due to the bound of 1 on $b$ and $c$. We can further restrict integration to the half-sector with $c<b$. In this sector, there remain 


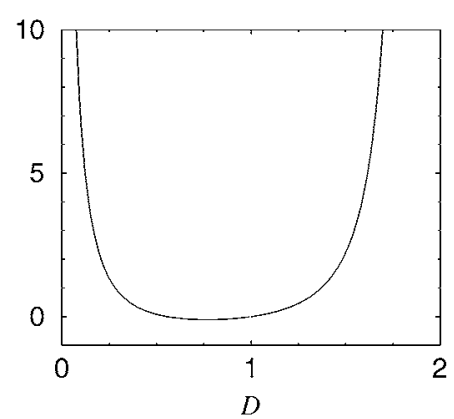

\begin{tabular}{|c|c|}
\hline $\mathrm{D}$ & \\
\hline 0.1 & 6.71 \\
0.2 & 2.11 \\
0.3 & 0.847 \\
0.4 & 0.325 \\
0.5 & 0.0807 \\
0.6 & -0.0415 \\
0.7 & -0.0949 \\
\hline
\end{tabular}

\begin{tabular}{|c|c|}
\hline $\mathrm{D}$ & \\
\hline 0.8 & -0.102 \\
0.9 & 0.0712 \\
1.0 & 0 \\
1.1 & 0.123 \\
1.2 & 0.321 \\
1.3 & 0.644 \\
1.4 & 1.20 \\
\hline
\end{tabular}

\begin{tabular}{|c|c|}
\hline $\mathrm{D}$ & \\
\hline 1.5 & 2.24 \\
1.6 & 4.45 \\
1.7 & 10.4 \\
1.8 & 36.7 \\
1.9 & 658 \\
2.0 & $\infty$ \\
& \\
\hline
\end{tabular}

Figure 6. Numerically obtained results for the two-loop diagram in the MS-scheme. Some values are tabulated.

singularities for small $\alpha$ and $c$. The reader is invited to verify that they are eliminated by a second change of variables

$$
\begin{aligned}
& \alpha=\frac{\pi}{2} \beta^{\frac{1}{D-1}} \\
& c=\tilde{c}^{\frac{1}{2-D}} .
\end{aligned}
$$

The above method works for $D>1$ only. For $D<1$, one has to analytically continue the integration over $c_{\perp}$ in (4.11). This can be done by partial integration, since the integrand does not explicitly depend on $c_{\perp}$ but on $c=\sqrt{c_{\perp}^{2}+c_{\|}^{2}}$. To have no boundary terms, one should partially integrate before mapping everything onto the sector $\mathcal{A}$. We have explicitly checked consistency with the earlier used measure for $1<D<2$. Note finally that in the special case of $D=1$ in (4.17) the integration measure becomes a distribution reducing the integration measure to a line along $c_{\|}$.

The diagram is shown in figure 6. It grows as $D^{-1}$ for $D \rightarrow 0$ and exponentially for $D \rightarrow 2$ :

$$
I_{2}(D) \sim\left(\frac{3}{4}\right)^{-d_{\mathrm{c}} / 2} \sim\left(\frac{3}{4}\right)^{-D /(2-D)} .
$$

In the case of polymers $D=1$ it vanishes. The reason is that the diagrams factorize, and thus the one-loop result is correct to all orders when working in an appropriate scheme (see e.g. [4]).

\section{3. $R G$-function and extrapolation}

From (3.27) the renormalization $\beta$-function in the second order of perturbation theory reads

$$
\beta(g)=-\varepsilon g+\frac{g^{2}}{2}-\frac{g^{3}}{3} I_{2}(D)+\mathrm{O}\left(g^{4}\right) .
$$

The long-distance behaviour of the theory is governed by the IR-stable fixed point of the RG-flow. Fixed points are given by zeros of the $\beta$-function:

$\beta\left(g^{*}\right)=0 \quad \Rightarrow \quad g^{*}=0 \vee-\varepsilon+\frac{g^{*}}{2}-\frac{g^{* 2}}{3} I_{2}(D)+\mathrm{O}\left(g^{* 3}\right)=0$.

The physically interesting nontrivial fixed point is the one that is next to zero. In an $\varepsilon$-expansion it reads

$$
g^{*}=2 \varepsilon+\frac{8}{3} I_{2}(D) \varepsilon^{2}+\mathrm{O}\left(\varepsilon^{3}\right)
$$

The correction to the scaling exponent $\omega$ at this fixed point is found in an $\varepsilon$-expansion to be

$$
\omega\left(g^{*}\right)=\varepsilon-\frac{4}{3} I_{2}(D) \varepsilon^{2}+\mathrm{O}\left(\varepsilon^{3}\right)
$$


$(\mathrm{D}, \mathrm{d})=(1,1)$

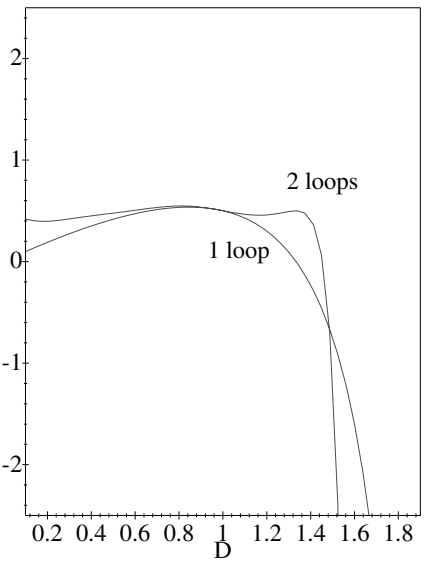

$(\mathrm{D}, \mathrm{d})=(1,1)$

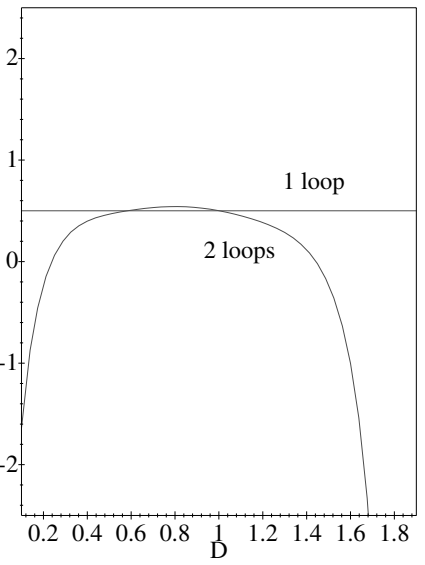

$(\mathrm{D}, \mathrm{d})=(1,1)$

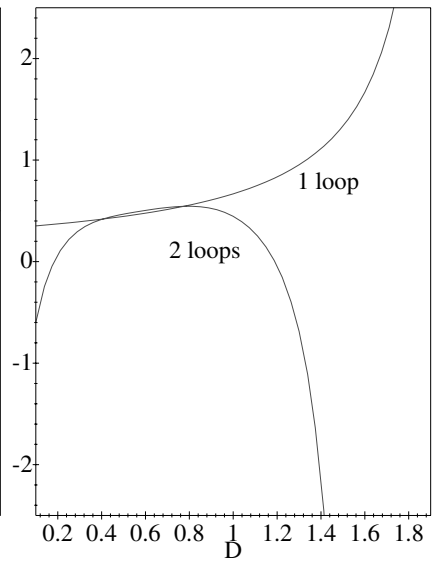

Figure 7. Correction to the scaling exponent $\omega$ for $(D=1, d=1)$ with different extrapolation parameters $(D, d),(D, \varepsilon(D, d)),\left(D, D_{\mathrm{c}}(d)\right)$ (from left to right). The abscissa labels the corresponding internal dimension $D_{0}$ of the departure point on the critical curve $\varepsilon\left(D_{0}, d_{0}\right)=0$.

The question now arises of how to calculate $\omega$ for a given physical situation. The general strategy that we follow in the MS-scheme is that we make use of the freedom to choose any point on the critical line (2.2) around which to start our expansion [30,31]. To expand towards some physically interesting point $(D, d)$ we furthermore dispose of the freedom to follow any path starting on the curve $\varepsilon=0$. Since our expansion (4.24) is exact in $D$ but only of second order in $\varepsilon$, we can equally well expand it to second order in $D$ around any point on the critical curve. Now we can change our extrapolation path through an invertible transformation $(x, y)=(x(D, d), y(D, d))$. One expresses $D$ and $\varepsilon$ as functions of $x$ and $y$ and reexpands $\omega$ to second order in $x$ and $y$ around the point $\left(x_{0}, y_{0}\right)=\left(x_{0}\left(D_{0}, d_{\mathrm{c}}\left(D_{0}\right)\right), y_{0}\left(D_{0}, d_{\mathrm{c}}\left(D_{0}\right)\right)\right)$, where we recall that $d_{\mathrm{c}}(D)$ is defined such that $\varepsilon\left(D, d_{\mathrm{c}}(D)\right)=0$. The aim is to find an optimal choice of variables $(x, y)$. The guidelines for such a choice have been discussed in [27], where this procedure has been successfully applied to extrapolate results for the anomalous dimension of selfavoiding membranes. As a general rule, we 'trust' most a result that is the least sensitive to the starting point of the extrapolation. Therefore, we would like to find a plateau for some suitable chosen variables $(x, y)$. This procedure works well for polymers, or more generally for membranes with inner dimension close to 1 . However it turns out that for membranes it works much less well than in the selfavoiding case: we could not find a plateau, but at best an extremum.

Some extrapolations are shown in the figures 7,8 . We start with polymers interacting with a $\delta$-wall corresponding to the point $(D, d)=(1,1)$. (Note that for $d<2$ the interaction becomes relevant for polymers.) This can be seen in figure 7 . The values obtained from the plateaus appearing at the two-loop level $(\omega \approx 0.55)$ are quite close to the exact value $\omega=1 / 2$, known from the fact that the result in $D=1$ at one-loop is exact. From the latter it follows that it is best to use $(D, \varepsilon)$ as extrapolation parameters and to stay in $D=1$.

Let us now turn to the results obtained for $\omega$ extrapolated to points $D=2$ (figure 8): the extrapolated value depends strongly on the starting-point on the critical line. We can identify a least sensitive value, which lies below $D=1$, reflecting the $D$-dependence of the two-loop diagram. We obtain identical results for different extrapolation paths. Note that if we fix $d=1$ and perform an $\varepsilon$-expansion in $D$ as is commonly done [32], then this corresponds to starting from $D_{\mathrm{c}}(d=1)=2 / 3<1$. The $d$-dependence of the extrapolated values is recovered in the massive scheme, which we discuss in the next section. 

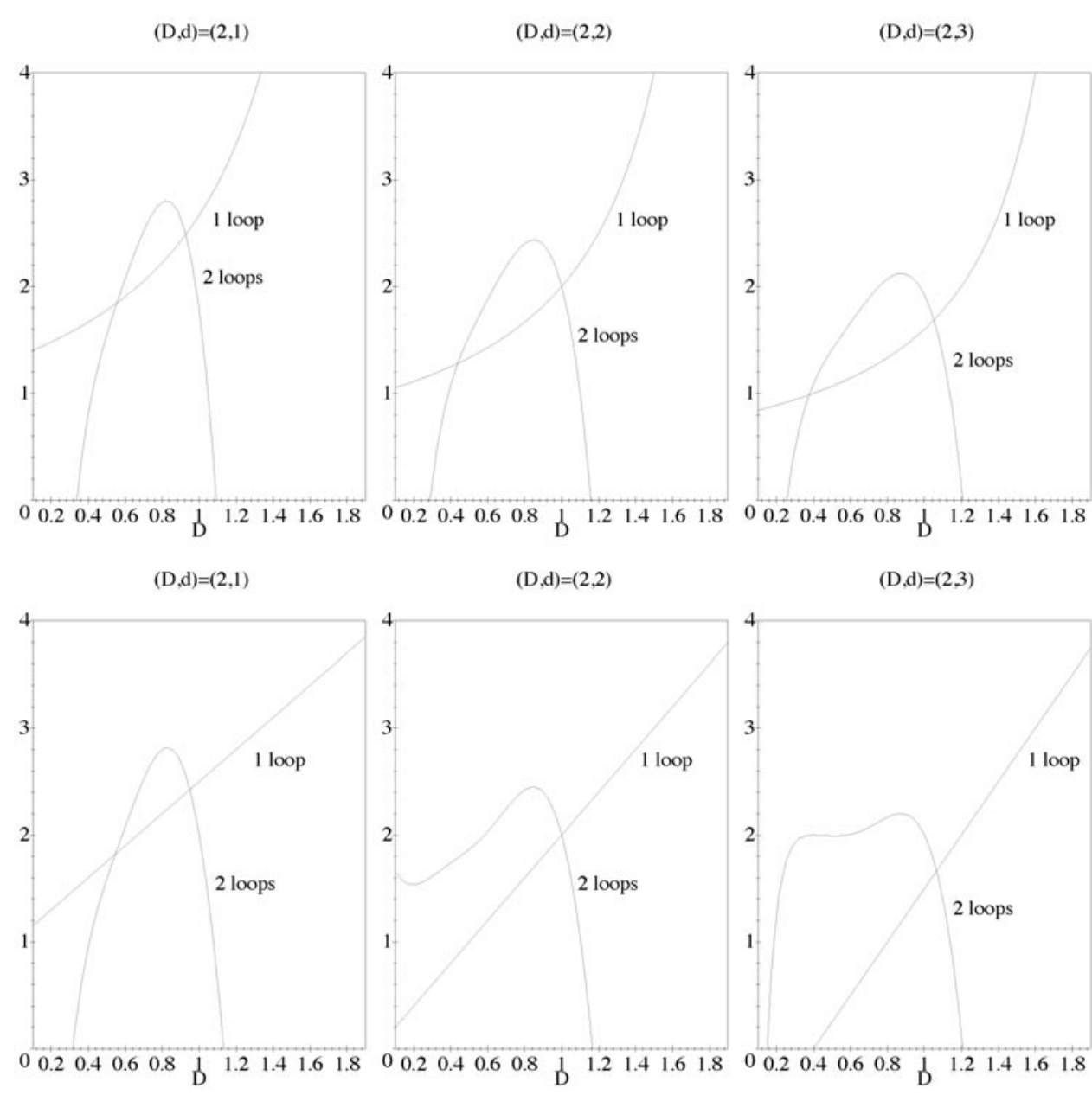

\begin{tabular}{|c|c|c|c|c|}
\hline$(x, y) ;(D, d)$ & $(1,1)$ & $(2,1)$ & $(2,2)$ & $(2,3)$ \\
\hline$(D, D c), \max$ & 0.55 & 2.80 & 2.43 & 2.10 \\
\hline$(D, d), \max$ & 0.55 & 2.80 & 2.43 & 2.20 \\
\hline$(D, \varepsilon), \max$ & 0.55 & & & \\
\hline
\end{tabular}

Figure 8. Correction to the scaling exponent $\omega$ for different extrapolation points $(D, d)$ : extrapolation parameters are $\left(D, D_{\mathrm{c}}(d)\right)$ (top) and $(D, d)$ (bottom). The abscissa labels the corresponding internal dimension $D_{0}$ of the departure point on the critical line $\varepsilon\left(D_{0}, d_{0}\right)=0$. Furthermore, the two-loop values with minimal sensitivity with respect to the departure point are tabulated.

\section{Calculation in fixed dimension: the massive scheme}

\subsection{Phantom manifolds}

An alternative scheme to an $\varepsilon$-expansion is a calculation in fixed dimension. This way we hope to circumvent the difficulty that we faced when calculating the two-loop diagram in the previous section in the limit of $D \rightarrow 2$ : Since the diagram behaves like $\left(\frac{4}{3}\right)^{d / 2}$, it becomes large when the dimension of the embedding space is large. When working in an $\varepsilon$-expansion, $d$ and 


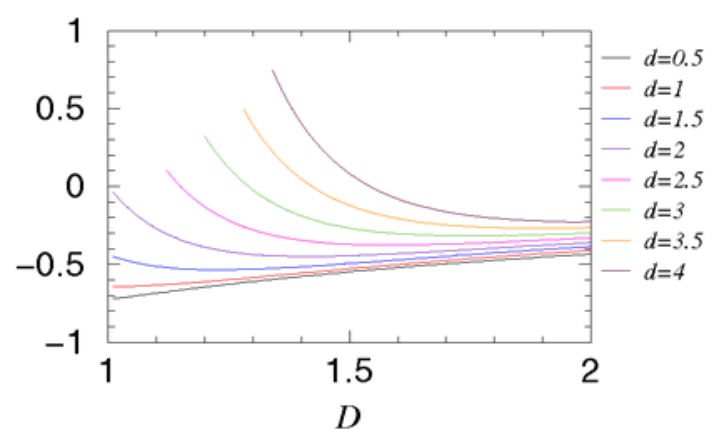

\begin{tabular}{|c|c|}
\hline$d$ & $I_{\left.2\right|_{D=2}}$ \\
\hline 0.5 & -0.437 \\
1 & -0.410 \\
1.5 & -0.386 \\
2 & -0.358 \\
2.5 & -0.331 \\
3 & -0.299 \\
3.5 & -0.265 \\
4 & -0.229 \\
\hline
\end{tabular}

Figure 9. Two-loop diagram in fixed dimension for embedding dimensions $d=0.5$ to 4 (from bottom to top). The curves are only shown for values of $D$ such that $\varepsilon \geqslant 0$.

$D$ are related by $\varepsilon=D-d(2-D) / 2$ and sending $\varepsilon \rightarrow 0$ before $D \rightarrow 2$ imposes that $d \rightarrow \infty$. Thus one would like to work at finite $d$, thus finite $\varepsilon$. The diagrams are UV convergent for $d<d_{\mathrm{c}}$. They are cut off in the infrared by a finite membrane size. Alternatively, we can use a 'soft' IR cutoff, that is we introduce a chemical potential $\tau$ into the Hamiltonian and sum over all manifold sizes. For polymers $(D=1)$ this scheme corresponds to a 'massive scheme' in an $O(N)$-symmetric, scalar $\phi^{4}$-theory as can be seen via a de Gennes-Laplace transformation on the $N=0$ component limit (the correlator in momentum-space reads $\frac{1}{k^{2}+t}$ ). In the following we keep a hard IR regulator and calculate the two-loop diagram in fixed dimension. We use the analytical expression given in (4.16). For the numerical integration in $1<D<2$ we proceed as before. The result for different embedding dimensions $d$ is shown graphically in figure 9, where also some explicit values in the limit of $D=2$ are given. Note that the exact factorization property in the limit of $D=1$ is only valid in the MS-scheme or in a massive scheme with suitable soft cutoff as mentioned above. However, even though the method is not tailored to capture that feature, the obtained value $\omega=0.6$ in the case of $(D, d)=(1,1)$ is not too far from the exact value $1 / 2$. We expect that higher-order terms would again reproduce the exact value of $\omega=1 / 2$. Also note that results for membranes $(D=2)$ are close to those from the $\varepsilon$-expansion, especially for small $d$.

\subsection{Selfavoiding membranes}

Up to now we have been considering a $D$-dimensional polymerized 'phantom' membrane interacting with a pointlike impurity in $d$-dimensional embedding space modelled through a $\delta$-potential. The infinite fractal dimension of the free manifold has been causing problems, and since all operators of the type $\left(-\Delta_{r}\right)^{n} \delta(r), n \geqslant 0$, attain the same relevance in $D=2$, it is not clear whether the model will break down. Physically, the problem is much better defined for selfavoiding membranes. From field-theoretical calculations, which have recently been refined up to two-loop [26,27], we know that two-dimensional selfavoiding manifolds embedded in three-dimensional space have an anomalous dimension of $v^{*} \approx 0.85$. We now try to overcome the problem of an infinite Hausdorff dimension by a crude simplification: we approximate the selfavoiding manifold by a Gaussian theory with the same scaling dimension, that is a Hamiltonian of the type [16]

$$
\mathcal{H}[r]=\frac{1}{k-D} \int_{x} \frac{1}{2} r(x)\left(-\Delta_{x}\right)^{k / 2} r(x)
$$


Table 1. Two-loop results for selfavoiding membranes $(D=2)$ approximated through a Gaussian theory having identical scaling dimension $v^{*}=0.85$. Results are shown for the corresponding diagram, the correction to the scaling exponent $\omega$ and the contact exponent $\theta$.

\begin{tabular}{rrrl}
\hline & \multicolumn{1}{c}{$I$} & $\omega\left(g^{*}\right)$ & $\theta\left(g^{*}\right)$ \\
\hline 1-loop & 1.000 & 1.15 & 2.71 \\
2-loop & -0.379 & 1.49 & 3.10 \\
\hline
\end{tabular}

where

$$
r: x \in \mathbb{R}^{D} \longrightarrow r(x) \in \mathbb{R}^{d}
$$

as before providing the two-point correlator in our normalizations:

$$
C(x, y)=|x-y|^{k-D} \text {. }
$$

The scaling dimension is

$$
v=\frac{k-D}{2}
$$

We recover (3.9) by setting $k=2$, but the model can be continued analytically to any real value of $k$, with $k \geqslant 2$. Setting

$$
k^{*}=2 v^{*}+D
$$

we get a Gaussian manifold with identical scaling dimension as obtained for selfavoiding crumpled membranes at two-loop. The critical embedding dimension of the interaction then reads

$$
d_{\mathrm{c}}=\frac{2 D}{k^{*}-D}=\frac{D}{v^{*}}
$$

that is the interaction is relevant in $d<d_{\mathrm{c}}$. For membranes $d_{\mathrm{c}}=2 / 0.85 \approx 2.4$. All operators of the type $\left(-\Delta_{r}^{n} \delta^{d}(r)\right)$ are at least naively irrelevant for $n>0$, as

$$
\left[\left(-\Delta_{r}^{n} \delta^{d}(r)\right)\right]=-v^{*}(d+2 n)
$$

and the corresponding coupling has in inverse length units the dimension

$$
\left[g_{0}^{(n)}\right]=D-v^{*}(d+2 n)<0 \quad n>0 .
$$

We may repeat the calculation in the massive scheme setting the free scaling dimension $v^{*}=0.85$ and $(D, d)=(2,1)$. The result is shown in table 1 .

Let us stress that this method cannot be turned into a systematic expansion, since perturbation theory neglects all effects of the non-Gaussian nature of the nontrivial crumpled state. We only know that for $d \rightarrow \infty$ the Gaussian variational approximation for the Hamiltonian, i.e. (5.1) becomes exact [33-35].

\section{Summation of the perturbation series in the limit $D=2$}

In the last section we introduced a 'massive' scheme allowing us to perform the renormalization procedure in fixed dimension. As can be seen in figures 9 and 10, the limit $D \rightarrow 2$ can be taken in the two-loop diagram and is smooth. Surprisingly, this limit can be calculated analytically: setting $v=0$ in (4.16), the integrand becomes constant, and thus trivial to calculate. This is a striking property. Even more, if we slightly modify the regularization prescription, this property holds to all loop orders in perturbation theory. Below, we shall give an analytic expression for the complete perturbation series, which allows for analysing the strong-coupling limit. 


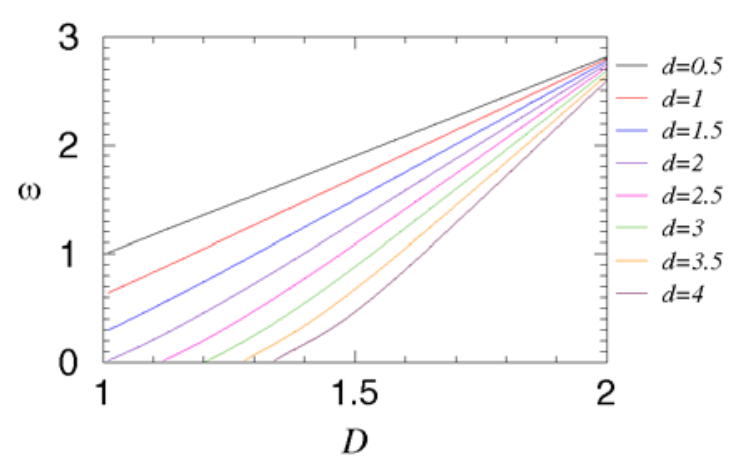

\begin{tabular}{|c|c|}
\hline$d$ & $\omega_{\left.\right|_{D=2}}$ \\
\hline 0.5 & 2.82 \\
1 & 2.80 \\
1.5 & 2.77 \\
2 & 2.75 \\
2.5 & 2.72 \\
3 & 2.69 \\
3.5 & 2.65 \\
4 & 2.60 \\
\hline
\end{tabular}

Figure 10. Correction to the scaling exponent $\omega$ for embedding dimension $d=0.5$ to 4 (from top to bottom). $\varepsilon$ is restricted to $\varepsilon \geqslant 0$; otherwise one has to work with an explicit UV cutoff.

Furthermore, we show how corrections in $(2-D)$ can be incorporated. We shall see that only the latter depend on the explicit cutoff procedure, a point which will be further clarified below. As a nontrivial check, when extrapolating back to polymers $(D=1)$, the corresponding result for $\omega$ is approximately reproduced.

\section{1. $N$-loop order}

In order to calculate the $N$-loop contribution to the perturbation theory we need the SDE of $N+1$ contact interactions. The normal-ordered product of the corresponding vertex operators reads

$$
\mathrm{e}^{\mathrm{i} k_{1} r\left(x_{1}\right)} \mathrm{e}^{\mathrm{i} k_{2} r\left(x_{2}\right)} \cdots \mathrm{e}^{\mathrm{i} k_{N+1} r\left(x_{N+1}\right)}=: \mathrm{e}^{\mathrm{i}\left(\sum_{n=1}^{N+1} k_{n} r\left(x_{n}\right)\right)}: \prod_{i, j=1}^{N+1} \mathrm{e}^{\frac{1}{2} k_{i} k_{j} C\left(x_{i}-x_{j}\right)} .
$$

We choose $x_{N+1}$ as root in the renormalization procedure (for the precise definition, see below) and therefore $k_{N+1}$ to integrate over in order to obtain the $\delta$-distribution (or its derivative), onto which to project. We thus shift

$$
k_{N+1} \longrightarrow k_{N+1}-\sum_{j=1}^{N} k_{j}
$$

and rewrite the quadratic form in (6.1) as

$$
\begin{aligned}
\frac{1}{2} \sum_{i, j=1}^{N+1} k_{i} k_{j} C\left(x_{i}-x_{j}\right)=\sum_{j=1}^{N} k_{N+1} k_{j} C\left(x_{N+1}-x_{j}\right)+\frac{1}{2} \sum_{i, j=1}^{N} k_{i} k_{j} C\left(x_{i}-x_{j}\right) \\
\longrightarrow \sum_{j=1}^{N} k_{N+1} k_{j} C\left(x_{N+1}-x_{j}\right)-\sum_{i, j=1}^{N} k_{i} k_{j} C\left(x_{N+1}-x_{j}\right) \\
+\frac{1}{2} \sum_{i, j=1}^{N} k_{i} k_{j} C\left(x_{i}-x_{j}\right)=\sum_{j=1}^{N} k_{N+1} k_{j} C\left(x_{N+1}-x_{j}\right) \\
-\sum_{i, j=1}^{N} k_{i} k_{j} \frac{C\left(x_{N+1}-x_{i}\right)+C\left(x_{N+1}-x_{j}\right)-C\left(x_{i}-x_{j}\right)}{2}
\end{aligned}
$$


Equation (6.1) then becomes (up to subdominant terms ${ }^{3}$ involving spatial derivatives of $r$ )

$$
: \mathrm{e}^{\mathrm{i} k_{N+1} r\left(x_{N+1}\right)}: \mathrm{e}^{\sum_{j=1}^{N} k_{N+1} k_{j} C\left(x_{N+1}-x_{j}\right)-\sum_{i, j=1}^{N} k_{i} k_{j} \frac{C\left(x_{N+1}-x_{i}\right)+C\left(x_{N+1}-x_{j}\right)-C\left(x_{i}-x_{j}\right)}{2}}
$$

and the integration over $k_{N+1}$ produces the $\delta$-interaction and its derivatives; the latter come from the first term in (6.2). They are subdominant and thus will be dropped. We abbreviate the quadratic form in (6.2) as $\left(D_{i j}\right)$, where the matrix elements are

$$
\begin{aligned}
& D_{i j}=\frac{1}{2}\left[C\left(x_{N+1}-x_{i}\right)+C\left(x_{N+1}-x_{j}\right)-C\left(x_{i}-x_{j}\right)\right] \quad i \neq j \\
& D_{i i}=C\left(x_{N+1}-x_{i}\right) .
\end{aligned}
$$

Integrating out the momenta $k_{1}, \ldots, k_{N}$ yields the operator product expansion of $N+1$ contact interactions as

$$
\begin{gathered}
\tilde{\delta}^{d}\left(r\left(x_{1}\right)\right) \tilde{\delta}^{d}\left(r\left(x_{2}\right)\right) \ldots \tilde{\delta}^{d}\left(r\left(x_{N+1}\right)\right)=\left[\operatorname{det}\left(D_{i j}\right)\right]^{-d / 2} \tilde{\delta}^{d}\left(r\left(x_{N+1}\right)\right) \\
+ \text { subdominant operators. }
\end{gathered}
$$

\subsection{The limit $D \rightarrow 2$ and $(2-D)$-expansion}

Whereas (6.5) is still completely general, we now want to study the limit $D \rightarrow 2$ as well as perturbations above it. We start by remarking that the matrix $\left(D_{i j}\right)$ may be expanded in powers of $2-D$ :

$$
D_{i j}=\sum_{n=0}^{\infty} \frac{(2-D)^{n}}{n !} D_{i j}^{(n)}
$$

where

$$
\begin{aligned}
& D_{i j}^{(n)}=\frac{\mu^{-2 v}}{2}\left(\ln ^{n}\left|\left(x_{N+1}-x_{i}\right) \mu\right|+\ln ^{n}\left|\left(x_{N+1}-x_{j}\right) \mu\right|-\ln ^{n}\left|\left(x_{i}-x_{j}\right) \mu\right|\right) \quad i \neq j \\
& D_{i i}^{(n)}=\mu^{-2 v} \ln ^{n}\left|\left(x_{N+1}-x_{i}\right) \mu\right|
\end{aligned}
$$

$\mu=1 / L$. Then,

$$
\begin{aligned}
\operatorname{det}(D) & =\mathrm{e}^{\operatorname{Tr} \ln \left(D_{i j}\right)} \\
& =\operatorname{det}\left(D^{(0)}\right) \mathrm{e}^{\operatorname{Tr} \ln \left[\mathbf{1}+\sum_{n=1}^{\infty} \frac{(2-D)^{n}}{n !}\left[D^{(0)}\right]^{-1} D^{(n)}\right]}
\end{aligned}
$$

where $\left[D^{(0)}\right]^{-1}$ denotes the inverse matrix to $D^{(0)}$. Let us expand the OPE-coefficient in (6.5) up to second order in $2-D$. Expanding first the logarithm,

$$
\begin{aligned}
\ln \left[\mathbf{1}+\sum_{n=1}^{\infty} \frac{(2-D)^{n}}{n !}\left[D^{(0)}\right]^{-1} D^{(n)}\right]=(2-D)\left[D^{(0)}\right]^{-1} D^{(1)} \\
+\frac{(2-D)^{2}}{2}\left[\left[D^{(0)}\right]^{-1} D^{(2)}-\left(\left[D^{(0)}\right]^{-1} D^{(1)}\right)^{2}\right]+\mathrm{O}\left((2-D)^{3}\right)
\end{aligned}
$$

and inserting into (6.8) one arrives at

$$
\begin{aligned}
{[\operatorname{det}(D)]^{-d / 2}=} & {\left[\operatorname{det}\left(D^{(0)}\right)\right]^{-d / 2}\left[1-\frac{(2-D) d}{2} \operatorname{Tr}\left[D^{(0)}\right]^{-1} D^{(1)}-\frac{(2-D)^{2} d}{4}\right.} \\
& \times\left[\operatorname{Tr}\left[D^{(0)}\right]^{-1} D^{(2)}-\operatorname{Tr}\left(\left[D^{(0)}\right]^{-1} D^{(1)}\right)^{2}\right. \\
& \left.\left.-\frac{d}{2} \operatorname{Tr}^{2}\left[D^{(0)}\right]^{-1} D^{(1)}\right]+\mathrm{O}\left((2-D)^{3}\right)\right] .
\end{aligned}
$$

${ }^{3}$ Here we argue in terms of an OPE. Note that in order to make these arguments rigorous, one should ask whether these terms affect the effective action at constant background field. This is not the case, since then $\nabla r=0$. 
The zeroth order is $\left[\operatorname{det}\left(D^{(0)}\right)\right]^{-d / 2}$. In order to proceed we need the latter and the inverse matrix $\left[D^{(0)}\right]^{-1}$. Let us first calculate the determinant. From (6.6) we have

$$
\begin{aligned}
D^{(0)} & =\mu^{-2 v}\left(\begin{array}{cccc}
1 & \frac{1}{2} & \ldots & \frac{1}{2} \\
\frac{1}{2} & 1 & \ddots & \vdots \\
\vdots & \ddots & \ddots & \frac{1}{2} \\
\frac{1}{2} & \ldots & \frac{1}{2} & 1
\end{array}\right) \\
& =\frac{\mu^{-2 v}}{2}\left(\left(\begin{array}{ccc}
1 & \ldots & 0 \\
\vdots & \ddots & \vdots \\
0 & \vdots & 1
\end{array}\right)+\left(\begin{array}{ccc}
1 & \ldots & 1 \\
\vdots & \ddots & \vdots \\
1 & \ldots & 1
\end{array}\right)\right)=\frac{\mu^{-2 v}}{2}(\mathbf{1}+N \boldsymbol{P}) \\
\boldsymbol{P} & =\frac{1}{N}\left(\begin{array}{ccc}
1 & \ldots & 1 \\
\vdots & \ddots & \vdots \\
1 & \ldots & 1
\end{array}\right)
\end{aligned}
$$

being the projector onto $(1,1, \ldots, 1)$. Denoting by $\operatorname{dim} \operatorname{Im}(\boldsymbol{P})$ the dimension of the space onto which $\boldsymbol{P}$ projects one has the general formula

$$
\operatorname{det}(\mathbf{1}+a \boldsymbol{P})=(1+a)^{\operatorname{dim} \operatorname{Im}(\boldsymbol{P})} .
$$

Since here $\operatorname{dim} \operatorname{Im}(\boldsymbol{P})=1$, we find

$$
\operatorname{det}\left(D^{(0)}\right)=\mu^{-2 v N} \frac{1+N}{2^{N}} .
$$

The inverse matrix of $(\mathbf{1}+N \boldsymbol{P})$ can be inferred from the ansatz

$$
(\mathbf{1}+N \boldsymbol{P})(\mathbf{1}+b \boldsymbol{P})=\mathbf{1} .
$$

Since $\boldsymbol{P}$ is a projector $\boldsymbol{P}^{2}=\boldsymbol{P}$ and this implies

$$
(N+b+N b) \boldsymbol{P}=\mathbf{0} .
$$

Therefore, $b=-N /(N+1)$ and

$$
\left[D^{(0)}\right]^{-1}=2\left(\mathbf{1}-\frac{N}{N+1} \boldsymbol{P}\right) \mu^{2 v} .
$$

To obtain the first-order contribution in $(2-D)$ from equation $(6.8)$ we only need $\left[D^{(0)}\right]^{-1} D^{(1)}$, which reads

$$
\left(\left[D^{(0)}\right]^{-1} D^{(1)}\right)_{i j}=\left(2 D_{i j}^{(1)}-\frac{2}{1+N} \sum_{k=1}^{N} D_{i k}^{(1)}\right) \mu^{2 v} .
$$

The trace of (6.13) can easily be performed, with the result

$\operatorname{Tr}\left(\left[D^{(0)}\right]^{-1} D^{(1)}\right)=\left(\frac{2 N}{1+N} \sum_{i=1}^{N} D_{i i}^{(1)}-\frac{2}{1+N} \sum_{i=1}^{N} \sum_{k=1}^{N}\left(1-\delta_{i k}\right) D_{i k}^{(1)}\right) \mu^{2 v}$.

In order to factorize the integrals as much as possible, we found it convenient to modify the regularization prescription (3.21). In each order of perturbation theory we have to integrate the expression (6.5) over internal distances. These integrals have to be regularized in the infrared through an appropriate IR cutoff. In the preceding sections we have cut off the integrals such that all relative distances between internal points were smaller than $L=1 / \mu$. In the following we replace this by the demand that all distances from the fixed, but arbitrarily chosen point $x_{N+1}$, the root of the subtraction prescription, are bounded by $L$. Other distances are not restricted (see figure 11). Alternatively, one could calculate on a closed manifold of finite size. 


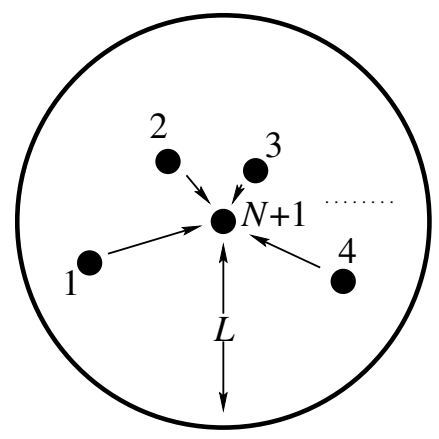

Figure 11. Regularization scheme for the $N$-loop diagrams.

Of course, then the correlator would have to be modified. Work is in progress to implement this procedure, which has the advantage of being more systematic [29].

To simplify the calculations, we further introduce the following notation:

$$
\overline{f\left(x_{i_{1}}, \ldots, x_{i_{k}}\right)}:=\int_{x_{1}} \ldots \int_{x_{N}} f\left(x_{i_{1}}, \ldots, x_{i_{k}}\right)
$$

with new normalization

$$
\int_{x}:=\frac{D}{S_{D}} \int \mathrm{d}^{D} x \theta(|x|<1)
$$

which is chosen such that the overbar can be thought of as an averaging procedure, and especially

$$
\overline{1}=1 .
$$

Thanks to our regularization prescription the integral of (6.14) over internal points can be replaced by $\mu^{-N D}$ (for the integration measure $L^{N D}$ ) times

$$
\begin{aligned}
\overline{\operatorname{Tr}\left(\left[D^{(0)}\right]^{-1} D^{(1)}\right)} & =\frac{2 N^{2}}{1+N} \overline{\ln \left|\left(x_{N+1}-x_{i}\right)\right|} \\
& -\left(\frac{2 N(N-1)}{1+N}\right)\left(\overline{\ln \left|x_{N+1}-x_{i}\right|}-\frac{1}{2} \overline{\ln \left|x_{i}-x_{j}\right|}\right) \\
= & \frac{2 N}{1+N} \overline{\ln \left|x_{N+1}-x_{i}\right|}+\frac{N(N-1)}{1+N} \overline{\ln \left|x_{i}-x_{j}\right|} .
\end{aligned}
$$

Introducing a diagrammatic notation

$$
\text { N+1 }:=\overline{\ln \left|x_{N+1}-x_{i}\right|}
$$

and

$$
\overbrace{\substack{N+1 \\ \mathrm{~N}_{\mathrm{m}}}}:=\overline{\ln \left|x_{j}-x_{i}\right|}
$$

the $N$-loop integral reads up to first order in $2-D$

$$
\begin{aligned}
\int(\operatorname{det} D)^{-d / 2} & =\mu^{-N \varepsilon}\left(\frac{1+N}{2^{N}}\right)^{-d / 2} \times\left[1-\frac{d}{2}(2-D)\left(\frac{2 N}{1+N} \sim \frac{N(N-1)}{1+N}\left(\sqrt{i_{0+1}^{N+1}}\right)\right)\right. \\
& \left.+\mathrm{O}\left((2-D)^{2}\right)\right] .
\end{aligned}
$$


We now turn to an explicit calculation of the diagrams. To first order in $2-D$ we have two contributions. The first (6.19) is

$$
\sim=\int_{x_{i}} \ln \left|x_{N+1}-x_{i}\right|=D \int_{0}^{1} \frac{\mathrm{d} x x^{D}}{x} \ln x=-\frac{1}{D} \longrightarrow-\frac{1}{2} \quad \text { as } \quad D \rightarrow 2 \text {. }
$$

The second diagram (6.20) is

$$
\overbrace{\mathrm{N}_{\mathrm{m}+1}}^{\mathrm{N}}=\frac{D^{2}}{S_{D}^{2}} \int_{0}^{1} \mathrm{~d}^{D} x_{i} \mathrm{~d}^{D} x_{j} \ln \left|\vec{x}_{i}-\vec{x}_{j}\right|
$$

where we have put vectors to stress that $x_{i}-x_{j}$ is not a scalar. It suffices to evaluate this in $D=2$. We find

$$
\left(\mathrm{i}_{\mathrm{N}+1}^{\mathrm{N}+1} \mathrm{~m}\right.
$$

Combining (6.21)-(6.23) we finally obtain for the renormalized coupling $g$ up to first order in $^{4} 2-D$

$$
\begin{aligned}
g=g_{0} \mu^{-\varepsilon}(1 & +\sum_{N=1}^{\infty} \frac{\left(-g_{0} 2^{d / 2} \mu^{-\varepsilon}\right)^{N}}{(N+1) !(1+N)^{d / 2}} \\
& \left.\times\left[1-(2-D) \frac{d}{2}\left(\frac{2 N}{1+N}\left(i^{N+1}\right)+\frac{N(N-1)}{1+N}\left(\mathrm{i}_{\mathrm{i}_{\mathrm{j}}+\mathrm{N}_{\mathrm{j}}}\right)\right)+\cdots\right]\right) \\
= & g_{0} \mu^{-\varepsilon}\left(1+\sum_{N=1}^{\infty} \frac{\left(-g_{0} 2^{d / 2} \mu^{-\varepsilon}\right)^{N}}{(N+1) !(1+N)^{d / 2}}\right. \\
\times & {\left.\left[1+(2-D) \frac{d}{2}\left(\frac{N}{1+N}+\frac{N(N-1)}{4(1+N)}\right)+\cdots\right]\right) . }
\end{aligned}
$$

This can also be written as

$$
\begin{gathered}
g 2^{d / 2}=-\sum_{N=1}^{\infty} \frac{\left(-g_{0} 2^{d / 2} \mu^{-\varepsilon}\right)^{N}}{N ! N^{d / 2}}-(2-D) \frac{d}{2} \sum_{N=1}^{\infty} \frac{\left(-g_{0} 2^{d / 2} \mu^{-\varepsilon}\right)^{N}(N-1)}{N ! N^{d / 2+1}} \\
-(2-D) \frac{d}{8} \sum_{N=1}^{\infty} \frac{\left(-g_{0} 2^{d / 2} \mu^{-\varepsilon}\right)^{N}(N-1)(N-2)}{N ! N^{d / 2+1}}
\end{gathered}
$$

Absorbing a factor of $2^{d / 2}$ both in $g$ and $g_{0}$ and defining the dimensionless coupling $z:=g_{0} \mu^{-\varepsilon}$, we obtain the final result

$$
\begin{gathered}
g=-\sum_{N=1}^{\infty} \frac{(-z)^{N}}{N ! N^{d / 2}}-(2-D) \frac{d}{2} \sum_{N=1}^{\infty} \frac{(-z)^{N}(N-1)}{N ! N^{d / 2+1}} \\
-(2-D) \frac{d}{8} \sum_{N=1}^{\infty} \frac{(-z)^{N}(N-1)(N-2)}{N ! N^{d / 2+1}}+\mathrm{O}(2-D)^{2} .
\end{gathered}
$$

This formula is the starting point for further analysis in the subsequent sections.

\subsection{Asymptotic behaviour of the series}

In the following we are interested in the limit of large $z$ (strong repulsion), which also is the scaling behaviour of infinitely large membranes. We therefore need an analytical expression for sums like (6.26) in the limit of large $z$. An example is the leading-order expression

$$
g=-\sum_{N=1}^{\infty} \frac{(-z)^{N}}{N ! N^{d / 2}}
$$

\footnotetext{
4 The expression in parenthesis is the inverse renormalization $Z_{g}$-factor which relates the bare coupling $g_{0}$ to the renormalized coupling $g$ according to $g Z_{g} \mu^{\varepsilon}=g_{0}$.
} 
For the following analysis we need an integral representation of the above series. We claim that for all $k, d>0$

$$
\sum_{N=0}^{\infty} \frac{(-z)^{N}}{N !(k+N)^{d / 2}}=\frac{1}{\Gamma\left(\frac{d}{2}\right)} \int_{0}^{\infty} \mathrm{d} r r^{d / 2-1} \mathrm{e}^{-z \mathrm{e}^{-r}-k r}
$$

This can be proven as follows:

$$
\begin{aligned}
\frac{1}{\Gamma\left(\frac{d}{2}\right)} \int_{0}^{\infty} \mathrm{d} r r^{d / 2-1} \mathrm{e}^{-z \mathrm{e}^{-r}-k r} & =\frac{1}{\Gamma\left(\frac{d}{2}\right)} \sum_{N=0}^{\infty} \frac{(-z)^{N}}{N !} \int_{0}^{\infty} \mathrm{d} r r^{d / 2-1} \mathrm{e}^{-(N+k) r} \\
& =\frac{1}{\Gamma\left(\frac{d}{2}\right)} \sum_{N=0}^{\infty} \frac{(-z)^{N}}{N !} \frac{\Gamma\left(\frac{d}{2}\right)}{(N+k)^{d / 2}}
\end{aligned}
$$

This integral representation is not the most practical for our purpose. It is better to set $r \rightarrow s:=\mathrm{e}^{-r}$, which yields

$$
\sum_{N=0}^{\infty} \frac{(-z)^{N}}{N !(k+N)^{d / 2}}=\frac{1}{\Gamma\left(\frac{d}{2}\right)} \int_{0}^{1} \mathrm{~d} s s^{k-1}(-\ln s)^{d / 2-1} \mathrm{e}^{-s z} .
$$

This formula is already very useful for some purposes. It is still advantageous to make a second variable-transformation $s \rightarrow y:=s z$, yielding

$$
\sum_{N=0}^{\infty} \frac{(-z)^{N}}{N !(k+N)^{d / 2}}=\frac{(\ln z)^{d / 2-1}}{\Gamma\left(\frac{d}{2}\right) z^{k}} \int_{0}^{z} \mathrm{~d} y y^{k-1}\left(1-\frac{\ln y}{\ln z}\right)^{d / 2-1} \mathrm{e}^{-y} .
$$

Finally we remark that we usually have the following combination:

$f_{k}^{d}(z):=z^{k} \sum_{N=0}^{\infty} \frac{(-z)^{N}}{N !(k+N)^{d / 2}}=\frac{(\ln z)^{d / 2-1}}{\Gamma\left(\frac{d}{2}\right)} \int_{0}^{z} \mathrm{~d} y y^{k-1}\left(1-\frac{\ln y}{\ln z}\right)^{d / 2-1} \mathrm{e}^{-y}$.

It satisfies the following simple recursion relation, which is helpful to calculate the $\beta$-function:

$$
z \frac{\mathrm{d}}{\mathrm{d} z} f_{k}^{d}(z)=f_{k}^{d-2}(z)
$$

From (6.28) $f_{k}(z)>0$ for all $k, d>0$ and the behaviour for large $z$ is obtained by expanding $\left(1-\frac{\ln y}{\ln z}\right)^{d / 2-1}$ for small $\frac{1}{\ln z}$

$$
\begin{gathered}
f_{k}^{d}(z)=\frac{(\ln z)^{d / 2-1}}{\Gamma\left(\frac{d}{2}\right)}\left[\int_{0}^{\infty} \mathrm{d} y y^{k-1} \mathrm{e}^{-y}-\frac{1}{\ln z}\left(\frac{d}{2}-1\right) \int_{0}^{\infty} \mathrm{d} y y^{k-1} \ln y \mathrm{e}^{-y}\right. \\
\left.+\mathrm{O}\left(\frac{1}{(\ln z)^{2}}\right)\right]+\mathrm{O}\left(\mathrm{e}^{-z}\right) .
\end{gathered}
$$

The result is

$$
f_{k}^{d}(z)=\frac{(\ln z)^{d / 2-1} \Gamma(k)}{\Gamma\left(\frac{d}{2}\right)}\left(1-\frac{1}{\ln z} \frac{d-2}{2} \frac{\Gamma^{\prime}(k)}{\Gamma(k)}+\cdots\right) .
$$

For later reference, we note a list of useful formulae $(\gamma=0.577216 \ldots$ is the Euler constant $)$

$$
\begin{aligned}
& f_{1}^{d}(z)=\frac{(\ln z)^{d / 2-1}}{\Gamma\left(\frac{d}{2}\right)}+\frac{\gamma(\ln z)^{d / 2-2}}{\Gamma\left(\frac{d-2}{2}\right)}+\frac{\left(6 \gamma^{2}+\pi^{2}\right)(\ln z)^{d / 2-3}}{12 \Gamma\left(\frac{d-4}{2}\right)}+\cdots \\
& f_{2}^{d}(z)=\frac{(\ln z)^{d / 2-1}}{\Gamma\left(\frac{d}{2}\right)}+\frac{(\gamma-1)(\ln z)^{d / 2-2}}{\Gamma\left(\frac{d-2}{2}\right)}+\frac{\left(6 \gamma(\gamma-2)+\pi^{2}\right)(\ln z)^{d / 2-3}}{12 \Gamma\left(\frac{d-4}{2}\right)}+\cdots \\
& f_{3}^{d}(z)=\frac{2(\ln z)^{d / 2-1}}{\Gamma\left(\frac{d}{2}\right)}+\frac{(2 \gamma-3)(\ln z)^{d / 2-2}}{\Gamma\left(\frac{d-2}{2}\right)}+\frac{\left(6 \gamma^{2}-18 \gamma+6+\pi^{2}\right)(\ln z)^{d / 2-3}}{6 \Gamma\left(\frac{d-4}{2}\right)}+\cdots
\end{aligned}
$$


Note that with the above notations, the relation (6.26) expressing $g$ as a function of $z$ becomes

$g(z)=f_{1}^{d+2}(z)-(2-D) \frac{d}{2} f_{2}^{d+4}(z)+(2-D) \frac{d}{8} f_{3}^{d+4}(z)+\mathrm{O}(2-D)^{2}$.

In the next two sections we shall present two formalisms which give results for $\omega$ at next to leading order. It will turn out that the results catch qualitatively correctly the cross-over to $D=1$; however, they differ in the numerical values. This is a reflection of the fact that the $(2-D)$-expansion is not systematic. We expect that working on a manifold of finite size and using the exact correlation function on this manifold would lead to a systematic expansion. Work is in progress [29] to check this hypothesis.

\subsection{The RG-functions in the bare coupling}

The renormalization group $\beta$-function is defined as

$$
\beta(g):=\left.\mu \frac{\mathrm{d}}{\mathrm{d} \mu}\right|_{0} g .
$$

Since it is hard to invert the function $g(z)$, it is advantageous to study $\beta$ as function of the bare coupling $z$

$$
\beta(g) \equiv \beta(z)=-\varepsilon z \frac{\mathrm{d}}{\mathrm{d} z} g .
$$

To first order in $2-D$, we find from (6.38) using (6.32)

$\beta(z)=-\varepsilon\left[f_{1}^{d}(z)+(2-D)\left(-\frac{d}{2} f_{2}^{d+2}(z)+\frac{d}{8} f_{3}^{d+2}(z)\right)+\mathrm{O}(2-D)^{2}\right]$.

Inserting the asymptotic expansions from the last section, this becomes

$$
\beta(z)=-\frac{\varepsilon}{\Gamma\left(\frac{d}{2}\right)}\left[(\ln z)^{d / 2-1}-\frac{2-D}{2}(\ln z)^{d / 2}+\cdots\right]
$$

where omitted terms have one more power of either $(2-D)$ or $1 / \ln z$. As shown in section 2 , universal properties are given by the correction to the scaling exponent $\omega$, reading

$$
\omega(z):=\frac{\mathrm{d} \beta(g)}{\mathrm{d} g}=\frac{\mathrm{d} \beta(z)}{\mathrm{d} z} \frac{1}{\frac{\mathrm{d} g}{\mathrm{~d} z}}=-\frac{\varepsilon z}{\beta(z)} \frac{\mathrm{d} \beta(z)}{\mathrm{d} z} .
$$

Let us first study $D=2$. $\beta(z)$ and $\omega(z)$ are plotted in figure 12 . We see that for $d<2$ the $\beta$-function becomes zero for $z \rightarrow \infty$. For $d>2, \beta(z)$ has no zero, but the flow of $g$ is still to infinity. In both cases $\omega$ is given by $\omega=\lim _{z \rightarrow \infty} \omega(z)$. Taking only the leading term in the $(2-D)$-expansion of $\beta(z), \omega(z)$ is given by

$$
\omega(z)=-\varepsilon \frac{z \frac{\mathrm{d}}{\mathrm{d} z} f_{1}^{d}(z)}{f_{1}^{d}(z)}=-\varepsilon \frac{d-2}{2 \ln z} \rightarrow 0 .
$$

Let us now take into account the first order in $2-D$. Then the fixed point of (6.42) is at the finite value

$$
\ln z^{*}=\frac{2}{2-D}
$$

Next, when trying to calculate $\omega(z)$, we face the following problem: since we truncated the series for $g$ at order one in $2-D, \beta^{\prime}(z)$ does not vanish at the fixed point, i.e. the zero of $\beta(z)$. This might lead to the conclusion that $\omega\left(z^{*}\right)$ is always $\infty$, an absurd result. However since 

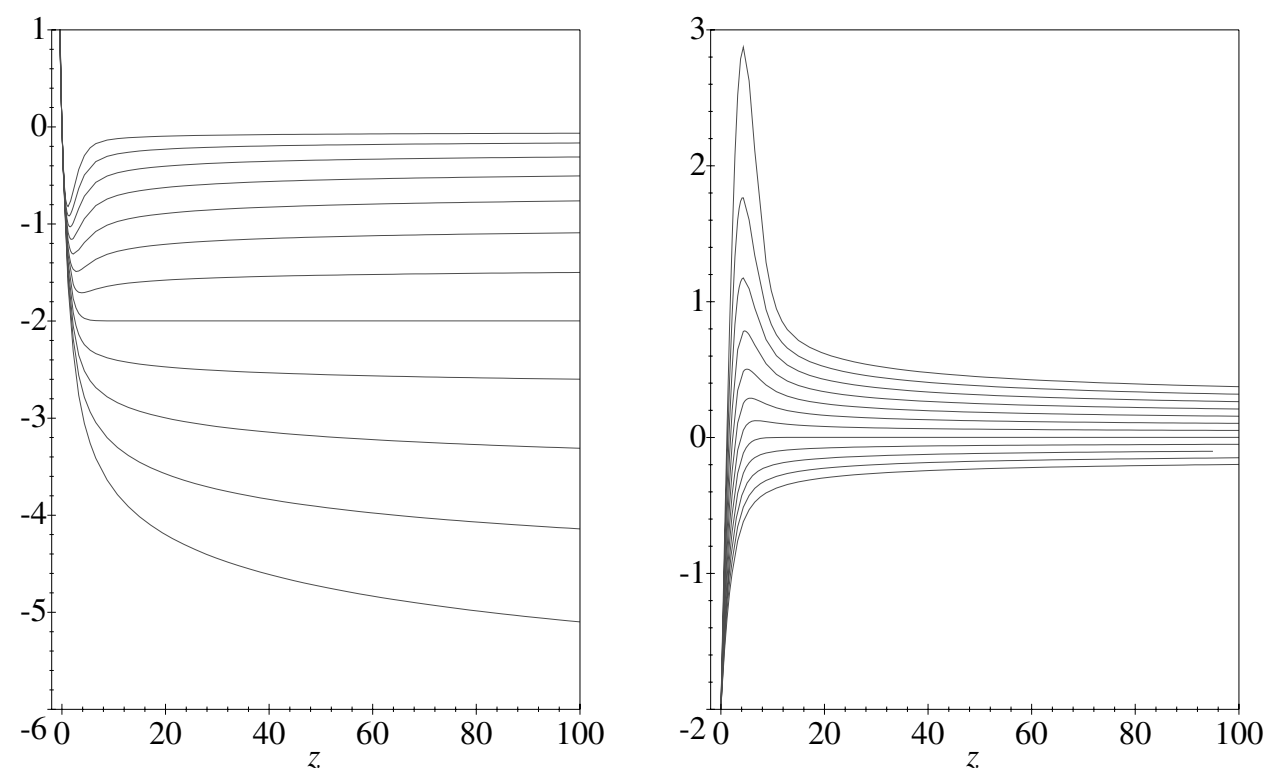

Figure 12. $\beta$-function (left) and $\omega$ (right) as functions of the dimensionless bare coupling $z$ for different dimensions $d: d=0.25,0.5,0.75, \ldots, 3$ (from top to bottom, respectively). Note that $\beta$ always has a fixed point at $z=0$ with $\omega(0)=-\varepsilon$. Furthermore, there is a fixed point for $0<d<2$ at $z=\infty$ with $\omega(\infty)=0$, the latter remaining true for $d \geqslant 2$. In $d=2 \beta$ tends to $-\varepsilon$ as $z \rightarrow \infty$. Above, $\beta$ diverges.

this is a consequence of the truncation of the series, we follow the strategy also to expand the denominator of $\omega(z)$ in powers of $2-D$. From (6.41) and (6.43) we obtain

$$
\begin{array}{r}
\omega(z)=-\varepsilon\left[\frac{z \frac{\mathrm{d}}{\mathrm{d} z} f_{1}^{d}(z)}{f_{1}^{d}(z)}+(2-D) \frac{z \frac{\mathrm{d}}{\mathrm{d} z}\left[-\frac{d}{2} f_{2}^{d+2}(z)+\frac{d}{8} f_{3}^{d+2}(z)\right]}{f_{1}^{d}(z)}\right. \\
\left.+(2-D) \frac{\left[\frac{d}{2} f_{2}^{d+2}(z)-\frac{d}{8} f_{3}^{d+2}(z)\right] z \frac{\mathrm{d}}{\mathrm{d} z} f_{1}^{d}(z)}{f_{1}^{d}(z)^{2}}\right] .
\end{array}
$$

Inserting the asymptotic series, we find

$$
\omega(z)=-\varepsilon \frac{d-2}{2 \ln z}+\varepsilon \frac{2-D}{2} .
$$

Inserting the fixed point (6.45), we arrive at

$$
\omega\left(z^{*}\right)=\varepsilon(2-D) \frac{4-d}{4} .
$$

This should be checked against the exact result in $D=1$, which reads

$$
\omega(D=1)=\varepsilon
$$

Thus our resummation procedure gives for $d \rightarrow 0$ the exact and for $d>0$ an approximative result. Before analysing the validity of the procedure, let us turn to the second scheme, namely the calculation in the renormalized coupling. 


\subsection{Calculation in the renormalized coupling}

We start from $g(z)$ given in (6.38). For large $z$ this can be approximated by

$$
g(z)=\frac{(\ln z)^{d / 2}}{\Gamma\left(\frac{d}{2}+1\right)}-(2-D) \frac{d}{4} \frac{(\ln z)^{d / 2+1}}{\Gamma\left(\frac{d}{2}+2\right)}
$$

where we only retained the leading term from each series. We shall focus here on $d<2$. Using (6.50), we can write $\ln z$ as a function of $g$. To first order in $2-D$, this reads

$$
\ln z=\tilde{g}^{2 / d}+\frac{2-D}{2+d} \tilde{g}^{4 / d} \quad \tilde{g}:=g \Gamma\left(\frac{d}{2}+1\right) .
$$

We now write the $\beta$-function in terms of $\tilde{g}$. Starting from (6.42) we obtain

$$
\beta(\tilde{g})=-\frac{\varepsilon}{\Gamma\left(\frac{d}{2}\right)}\left[\tilde{g}^{1-2 / d}-\frac{2(2-D)}{2+d} \tilde{g}\right] .
$$

Fixed points are at the zeros of the $\beta$-function. The nontrivial one is at

$$
\tilde{g}^{*}=\left[\frac{2+d}{2(2-D)}\right]^{d / 2} \text {. }
$$

The correction to the scaling exponent $\omega$ is simply obtained by evaluating the derivative of $\beta$ with respect to $g$ at the fixed point. In terms of $\tilde{g}$ this is

$$
\begin{aligned}
\omega(\tilde{g}) & =\frac{\mathrm{d} \beta(g)}{\mathrm{d} g}=\frac{\mathrm{d} \beta(\tilde{g})}{\mathrm{d} \tilde{g}} \frac{\mathrm{d} \tilde{g}}{\mathrm{~d} g}=\Gamma\left(\frac{d}{2}+1\right) \frac{\mathrm{d} \beta(\tilde{g})}{\mathrm{d} \tilde{g}} \\
& =-\varepsilon\left[\left(\frac{d}{2}-1\right) \tilde{g}^{-2 / d}-\frac{d(2-D)}{2+d}\right] .
\end{aligned}
$$

Inserting the fixed point $\tilde{g}^{*}$ from (6.53) we find

$$
\omega\left(\tilde{g}^{*}\right)=\varepsilon \frac{2(2-D)}{2+d} .
$$

Again, this is quite close to the exact result $\omega(D=1)=\varepsilon$ in $D=1$, and in effect exact in $D=1$ and $d=0$.

Let us finally point out that in the limit $D=2$ the true asymptotic behaviour of the $\beta$-function in terms of the renormalized coupling $g$ is obtained from the completely summed series (6.27) leading to (6.52) for large $g$. Conversely, if one tries to invert (6.27) and truncates it taking only a finite number of orders into account, it is at least possible to reach the asymptotic regime-however, for large enough $g$ the truncated $\beta$-function wildly oscillates and thus strongly deviates from the true behaviour. In figure 13 the Pade-resummed truncated $\beta$ function up to order $g^{160}$ in $d=1$ is compared with the exact, asymptotic flow-function. One notices that the truncated $\beta$-function, even though improved through a Pade resummation, hardly gets into touch with the asymptotic regime. The same applies to the slope-function $\omega(g)$, which is not shown in figure 13 .

Note that the above arguments suggest quite intuitively the behaviour of the exact $\beta$ function in $1<D<2$. Whereas for $D=1$ the $\beta$-function is a parabola, and for $D=2$ it decays like a power-law for large $g$ at least as long as $d<2$, the $\beta$-function for values of $D$ between these two extremes should cut the axis $\beta(g)=0$ at a finite value of $g$, which for $D \rightarrow 2$ wanders off to infinity, thus by continuity forcing the exponent $\omega$ to go to zero for $D \rightarrow 2$. 


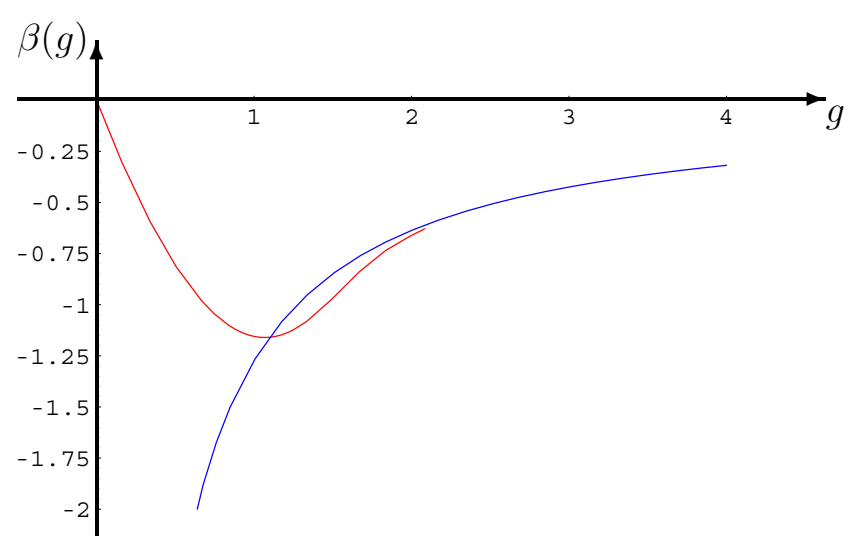

Figure 13. $\beta$-function in terms of the renormalized coupling $g$ truncated at order 160, Pade resummed and plotting only that part for which the truncated series converges. (This can be tested, e.g. by taking away the last few terms of the series.) This is compared with the asymptotic behaviour (6.52) (proportional to $1 / g$ for large $g$ ). $d$ is set to unity, and we used the diagonal ( 80 , 80)-Pade approximant, which was found to converge best. (The nonresummed expression starts to diverge already at $g \approx 1.8$ at this order.)

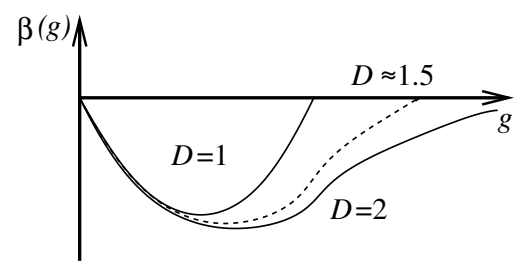

Figure 14. Qualitative behaviour for the $\beta$-function in $D=1,2$ and result anticipated for $D \approx 1.5$.

\section{Discussion and conclusion}

In this paper, we have discussed the perturbation expansion of a $D$-dimensional elastic manifold interacting via a $\delta$-interaction with a fixed point in embedding space. This calculation can be performed exactly for $D=1$, but becomes nontrivial for $D \neq 1$. Interestingly and quite surprisingly, it simplifies considerably in the limit of $D \rightarrow 2$, if one decides to work at finite $\varepsilon$. In that limit we were able to obtain an explicit expression for the renormalized coupling as a function of the bare one, in terms of a nontrivial series. Analysis of this series shows that the fixed point lies at infinity in the bare coupling, a limit in which we were able to derive an asymptotic expansion for the renormalized coupling as a function of the bare one. This yields a vanishing exponent $\omega$ in the limit of $D \rightarrow 2$. Also it is important to note that this result is completely independent of the regularization procedure. This no longer holds true beyond the leading order, which should be accessible to an expansion in $(2-D)$. We here constructed its first order in a specific regularization scheme. While this reproduces qualitatively correctly the known results in $D=1$ (and even exactly for $d=0$ ), it also shows by its dependence on the renormalization scheme (here working with the $\beta$-function either in terms of the renormalized or bare coupling) that this expansion in $(2-D)$ is not systematic. Even though experience with this new kind of expansion is still lacking, this is likely to be caused by the use of a hard cutoff in position space while working with the correlator of an infinite membrane. It seems that only in an $\varepsilon$-expansion is this procedure systematic. Work is in progress [29] to study the 
model on a sphere or torus of finite size, such that no further infrared cutoff is necessary. This should lead to exact results beyond the leading order and provide for the crossover between polymers and membranes.

While results for the pinning problem are interesting on their own, the main motivation is certainly to obtain a better understanding of selfavoiding polymerized membranes. This model is well behaved physically, since its fractal dimension $d_{\mathrm{f}}$ is bounded by the dimension of the embedding space $d_{\mathrm{f}}<d$. Preliminary studies [29] indicate that this problem can also be attacked by the methods developed in this paper. This would be very welcome to check the two-loop calculations [26,27] and the large-order behaviour [36] on one hand and numerical results (e.g. [28]) on the other.

\section{Acknowledgments}

It is a pleasure to thank R Blossey, F David, H W Diehl, M Kardar and L Schäfer for useful discussions. The clarity of this presentation has profited from a critical reading by $\mathrm{H}$ W Diehl. We are grateful to Andreas Ludwig for persistent questions, and his never tiring efforts to understand the limit of $D \rightarrow 2$. This work has been supported by the DFG through the Leibniz program Di 378/2-1, under Heisenberg grant Wi 1932/1-1, and NSF grant PHY99-07949.

\section{Appendix A. Universal 1/r-repulsion law for polymers}

We have shown in section 2.2 by a scaling argument that as long as $r \ll L^{v}$, the restricted partition function of a manifold pinned at one of its internal points scales as $\mathcal{Z}_{\infty}\left(r / L^{\nu}\right) \sim$ $\left(r / L^{\nu}\right)^{\theta}$, with the contact-exponent given by (2.25). It is instructive to prove this for the special case of polymers in $d=1$. According to (2.1) and (2.6) this corresponds to a polymer in $3 \mathrm{D}$-space interacting with a $\delta$-potential on a plane.

Let us start from the (bulk) polymer propagator being defined as the conditional probability of finding the internal point $s^{\prime}$ at the position $r_{\mathrm{f}}$ when starting in $s$ at $r_{\mathrm{i}}$. The propagator can be written as a functional integral for a restricted partition function of the free chain according to

$$
\begin{aligned}
\mathcal{Z}_{s, s^{\prime}}\left(r_{\mathrm{i}} \mid r_{\mathrm{f}}\right) & =\int \mathcal{D}[r] \tilde{\delta}\left(r(s)-r_{\mathrm{i}}\right) \tilde{\delta}\left(r\left(s^{\prime}\right)-r_{\mathrm{f}}\right) \mathrm{e}^{-\mathcal{H}[r]} \\
& =\int_{k} \mathrm{e}^{-k^{2}\left|s^{\prime}-s\right|+\mathrm{i} k\left(r_{\mathrm{f}}-r_{\mathrm{i}}\right)}=\left|s^{\prime}-s\right|^{-d / 2} \mathrm{e}^{-\left(r_{\mathrm{f}}-r_{\mathrm{i}}\right)^{2} / 4\left|s^{\prime}-s\right|} .
\end{aligned}
$$

The propagator (A.1) possesses the Markov property

$$
\mathcal{Z}_{s, s^{\prime \prime}}\left(r_{\mathrm{i}} \mid r_{\mathrm{f}}\right)=\int \mathrm{d}^{d} r_{m} \mathcal{Z}_{s, s^{\prime}}\left(r_{\mathrm{i}} \mid r_{m}\right) \mathcal{Z}_{s^{\prime}, s^{\prime \prime}}\left(r_{m} \mid r_{\mathrm{f}}\right)
$$

In the following we are interested in the restricted partition function (A.1) in the presence of a short-range interaction modelled through a $\delta$-potential, which is situated at the origin of the embedding space. The restricted partition function in the presence of the interaction will be denoted by $\mathcal{Z}_{s, s^{\prime}}^{g_{0}}\left(r_{\mathrm{i}} \mid r_{\mathrm{f}}\right)$. We consider the case where the chain is fixed at its ends, such that $s=0$ and $s^{\prime}=L$. We furthermore switch to a grand-canonical ensemble and denote

$$
\mathcal{Z}_{\tau}^{g_{0}}\left(r_{\mathrm{i}} \mid r_{\mathrm{f}}\right):=\int_{0}^{\infty} \mathrm{d} L \mathcal{Z}_{0, L}^{g_{0}}\left(r_{\mathrm{i}} \mid r_{\mathrm{f}}\right) \mathrm{e}^{-\tau L}
$$

$\tau$ being some chemical potential. $\mathcal{Z}_{\tau}^{g_{0}}\left(r_{\mathrm{i}} \mid r_{\mathrm{f}}\right)$ can be expanded in a perturbation series in the coupling $g_{0}$ according to

$$
\mathcal{Z}_{\tau}^{g_{0}}\left(r_{\mathrm{i}} \mid r_{\mathrm{f}}\right)=\sum_{n=0}^{\infty}\left(-g_{0}\right)^{n} \mathcal{Z}_{\tau}^{g_{0}, n}\left(r_{\mathrm{i}} \mid r_{\mathrm{f}}\right) .
$$


The $\mathcal{Z}_{\tau}^{g_{0}, n}\left(r_{\mathrm{i}} \mid r_{\mathrm{f}}\right)$ are

$$
\begin{aligned}
\mathcal{Z}_{\tau}^{g_{0}, n}\left(r_{\mathrm{i}} \mid r_{\mathrm{f}}\right):= & \int_{0}^{\infty} \mathrm{d} L \int_{0<x_{1}<\cdots<x_{n}<L} \mathcal{Z}_{0, x_{1}}\left(r_{\mathrm{i}} \mid 0\right) \mathcal{Z}_{x_{1}, x_{2}}(0 \mid 0) \\
& \times \cdots \times \mathcal{Z}_{x_{n-1}, x_{n}}(0 \mid 0) \mathcal{Z}_{x_{n}, L}\left(0 \mid r_{\mathrm{f}}\right) \mathrm{e}^{-\tau L} \\
= & {\left[\int_{0}^{\infty} \mathrm{d} x \mathrm{e}^{-x \tau} \mathcal{Z}_{0, x}\left(r_{\mathrm{i}}, 0\right)\right]\left[\int_{0}^{\infty} \mathrm{d} x \mathrm{e}^{-x \tau} \mathcal{Z}_{0, x}(0,0)\right]^{n-1} } \\
& \times\left[\int_{0}^{\infty} \mathrm{d} x \mathrm{e}^{-x \tau} \mathcal{Z}_{0, x}\left(0, r_{\mathrm{f}}\right)\right] \\
= & \mathcal{Z}_{\tau}^{0}\left(r_{\mathrm{i}} \mid 0\right) \mathcal{Z}_{\tau}^{0}(0 \mid 0)^{n-1} \mathcal{Z}_{\tau}^{0}\left(0 \mid r_{\mathrm{f}}\right)
\end{aligned}
$$

where we have used the fact that after integration over $L$, the integrals factorize; we further have defined

$$
\begin{aligned}
\mathcal{Z}_{\tau}\left(r_{\mathrm{i}} \mid r_{\mathrm{f}}\right) & =\int_{0}^{\infty} \mathrm{d} s \mathcal{Z}_{0, s}\left(r_{\mathrm{i}} \mid r_{\mathrm{f}}\right) \mathrm{e}^{-\tau s} \\
& =\int_{0}^{\infty} \mathrm{d} s \int_{k} \mathrm{e}^{-\left(k^{2}+\tau\right) s+\mathrm{i} k\left(r_{\mathrm{f}}-r_{\mathrm{i}}\right)} \\
& =\int_{k} \frac{\mathrm{e}^{\mathrm{i} k\left(r_{\mathrm{f}}-r_{\mathrm{i}}\right)}}{k^{2}+\tau}
\end{aligned}
$$

In $d=1$ the integration over the momenta $k$ in (A.6) leads to

$$
\mathcal{Z}_{\tau}\left(r_{\mathrm{i}} \mid r_{\mathrm{f}}\right)_{\mid d=1}=\sqrt{\frac{\pi}{\tau}} \mathrm{e}^{-\sqrt{\tau}\left|r_{\mathrm{f}}-r_{\mathrm{i}}\right|} .
$$

Let us now look at what terms appear in the $n$ th-order coefficient in (A.5): from the initial point of the chain a factor $\mathcal{Z}_{\tau}\left(r_{\mathrm{i}} \mid 0\right)$ is contributed, while the final point comes up with $\mathcal{Z}_{\tau}\left(0 \mid r_{\mathrm{f}}\right)$. Furthermore, the internal points give $n-1$ powers of $\mathcal{Z}_{\tau}(0 \mid 0)$, where

$$
\mathcal{Z}_{\tau}(0 \mid 0)=\sqrt{\frac{\pi}{\tau}}
$$

The $(n=0)$-coefficient is special: clearly, one has

$$
\mathcal{Z}_{\tau}^{g_{0}, 0}\left(r_{\mathrm{i}} \mid r_{\mathrm{f}}\right)=\mathcal{Z}_{\tau}\left(r_{\mathrm{i}} \mid r_{\mathrm{f}}\right)
$$

Inserting (A.8) and (A.9) into (A.5) we obtain for (A.4)

$$
\begin{array}{r}
\mathcal{Z}_{\tau}^{g_{0}}\left(r_{\mathrm{i}} \mid r_{\mathrm{f}}\right)=\sqrt{\frac{\pi}{\tau}}\left[\mathrm{e}^{-\sqrt{\tau}\left|r_{\mathrm{f}}-r_{\mathrm{i}}\right|}+\mathrm{e}^{-\sqrt{\tau}\left(\left|r_{\mathrm{f}}\right|+\left|r_{\mathrm{i}}\right|\right)} \sum_{n=1}^{\infty}\left(-g_{0} \sqrt{\frac{\pi}{\tau}}\right)^{n}\right] \\
=\sqrt{\frac{\pi}{\tau}}\left[\mathrm{e}^{-\sqrt{\tau}\left|r_{\mathrm{f}}-r_{\mathrm{i}}\right|}-\mathrm{e}^{-\sqrt{\tau}\left(\left|r_{\mathrm{f}}\right|+\left|r_{\mathrm{i}}\right|\right)} \frac{1}{1+\frac{1}{g_{0}} \sqrt{\frac{\tau}{\pi}}}\right] .
\end{array}
$$

The limit of $g_{0} \rightarrow \infty$ can be taken and leaves us with the beautiful result

$$
\mathcal{Z}_{\tau}^{\infty}\left(r_{\mathrm{i}} \mid r_{\mathrm{f}}\right)=\sqrt{\frac{\pi}{\tau}}\left(\mathrm{e}^{-\sqrt{\tau}\left|r_{\mathrm{f}}-r_{\mathrm{i}}\right|}-\mathrm{e}^{-\sqrt{\tau}\left(\left|r_{\mathrm{f}}\right|+\left|r_{\mathrm{i}}\right|\right)}\right) .
$$

This is the propagator of a scalar field with Dirichlet boundary conditions. It has applications when studying critical phenomena of for example an Ising magnet in half-space [37, 38]. Performing the inverse Laplace-transformation of (A.11) we arrive at

$$
\mathcal{Z}_{L}^{\infty}\left(r_{\mathrm{i}} \mid r_{\mathrm{f}}\right)=\frac{1}{L^{1 / 2}}\left(\mathrm{e}^{-\left(r_{\mathrm{f}}-r_{\mathrm{i}}\right)^{2} / 4|L|}-\mathrm{e}^{-\left(\left|r_{\mathrm{f}}\right|+\left|r_{\mathrm{i}}\right|\right)^{2} / 4|L|}\right)
$$


We conclude that a $\delta$-potential acting in a hyper-plane suppresses all configurations which penetrate or touch it, when taking its amplitude to infinity. Finally, in order to prove the universal repulsion law, we start from (A.12) and integrate over all final positions $r_{\mathrm{f}}$ :

$$
\mathcal{Z}_{L}^{\infty}\left(r_{\mathrm{i}}\right)=\int_{-\infty}^{\infty} \mathrm{d} r_{\mathrm{f}} \mathcal{Z}_{L}^{\infty}\left(r_{\mathrm{i}} \mid r_{\mathrm{f}}\right)=2 \sqrt{\pi}\left(1-\operatorname{erf} \frac{\left|r_{\mathrm{i}}\right|}{2 \sqrt{L}}\right)
$$

erf denoting the error function. For small arguments we have

$$
1-\operatorname{erf}(x) \sim x .
$$

Furthermore, $\mathcal{Z}_{L}^{\infty}\left(r_{\mathrm{i}}\right)$ denotes the restricted partition function of a chain pinned at one of its ends in $r_{\mathrm{i}}$. To obtain $\mathcal{Z}_{\infty}\left(r / L^{\nu}\right)$ in (2.23) we have to evaluate

$$
\mathcal{Z}_{\infty}\left(r / L^{v}\right)=\frac{1}{L} \int_{0}^{L} \mathrm{~d} s \mathcal{Z}_{s}^{\infty}(r) \mathcal{Z}_{L-s}^{\infty}(r) \sim\left(\frac{r}{\sqrt{L}}\right)^{2}
$$

according to (A.14) in the scaling regime $r \ll L^{1 / 2}$. We thus find that $\theta=2$, which confirms (2.25) for $D=1$ and $d=1$. The above proof can be extended to arbitrary dimensions $0<d<2$, where $\varepsilon>0$ for polymers. Then, $\theta=4-2 d$.

It would be nice to make the same arguments for membranes. However, the proof is based on a drastic simplification, which only occurs in $D=1$ and shows up in the factorizability of loop diagrams as in (A.5). This has no extension to manifolds of internal dimension $D>1$.

\section{Appendix B. Conformal mapping of the sectors}

In order to calculate the two-loop diagram efficiently, one wants to write it as an integral over a finite domain only. To do so we need the technique of conformal mapping of the sectors, which also serves for analytically continuing the measure of integration to internal dimensions $D<1$. This technique has been extensively used and well documented in the context of selfavoiding tethered membranes $[4,27,30]$, but we repeat the presentation here for completeness.

Generally, in evaluating the three-point divergences we need to integrate over some domain in the upper half-plane (see (4.11)). The measure of integration reads

$$
\int_{y}=\frac{S_{D-1}}{S_{D}} \int_{-\infty}^{\infty} \mathrm{d} y_{1} \int_{0}^{\infty} \mathrm{d} y_{2}\left(y_{2}\right)^{D-2}
$$

and the integrand is a function $f$ of the three distances $a, b$ and $c$ between the points $O=(0,0)$, $H=(-L, 0)$ and $Y=\left(y_{1}, y_{2}\right)$, given explicitly by (see figure B.1)

$a=L=$ fixed $\quad b=\sqrt{\left(y_{1}\right)^{2}+\left(y_{2}\right)^{2}} \quad c=\sqrt{\left(L-y_{1}\right)^{2}+\left(y_{2}\right)^{2}}$.

In our problem $f$ is homogeneous, of degree $\lambda$, but not necessarily symmetric:

$$
f(\kappa a, \kappa b, \kappa c)=\kappa^{-\lambda} f(a, b, c) .
$$

Let us now explicitly show how sectors can be mapped onto each other. Specializing to the mapping $\mathcal{B} \rightarrow \mathcal{A}$ we choose a coordinate system as given in figure B.1. The mapping $\mathcal{B} \rightarrow \mathcal{A}$ is mediated by a special conformal transformation, the inversion with respect to the circle around $\mathrm{O}$ and radius $L$. In complex coordinates this is ( $\bar{Y}$ is the complex conjugate of $Y$ )

$$
Y \longrightarrow \tilde{Y}=\frac{L^{2}}{\bar{Y}}=Y \frac{L^{2}}{b^{2}} \quad \Leftrightarrow \quad Y=\tilde{Y} \frac{L^{2}}{\tilde{b}^{2}}
$$

such that

$$
\left(\begin{array}{l}
y_{1} \\
y_{2}
\end{array}\right) \longrightarrow\left(\begin{array}{c}
\tilde{y}_{1} \\
\tilde{y}_{2}
\end{array}\right)=\left(\begin{array}{c}
\frac{y_{1} L^{2}}{b^{2}} \\
\frac{y_{2} L^{2}}{b^{2}}
\end{array}\right) \quad \Leftrightarrow \quad\left(\begin{array}{c}
y_{1} \\
y_{2}
\end{array}\right)=\left(\begin{array}{c}
\frac{\tilde{y}_{1} L^{2}}{\tilde{b}^{2}} \\
\frac{\tilde{y}_{2} L^{2}}{\tilde{b}^{2}}
\end{array}\right)
$$




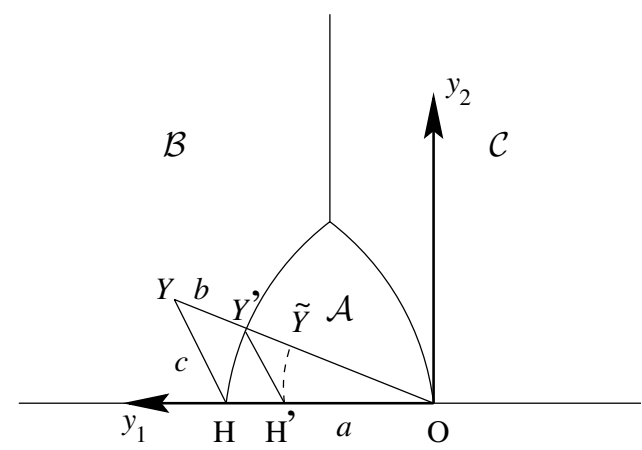

Figure B.1. The sectors $\mathcal{A}, \mathcal{B}$ and $\mathcal{C}$.

This change of coordinates gives a Jacobian for the measure (B.1)

$$
\left(\frac{L^{2}}{\tilde{b}^{2}}\right)^{D}
$$

It can easily be seen that the mapping $\mathcal{B} \rightarrow \mathcal{A}$ is one to one. First, let $Y=\left(y_{1}, y_{2}\right) \in \mathcal{B}$, that is $b>L$ and $b>c$. Then,

$$
\tilde{c}^{2}=\left(\left(\tilde{y}_{1}-L\right)^{2}+\left(\tilde{y}_{2}\right)^{2}\right)=\frac{L^{4}-2 y_{1} L^{3}+b^{2} L^{2}}{b^{2}}=L^{2} \frac{c^{2}}{b^{2}}<L^{2}
$$

and

$$
\tilde{b}^{2}=L^{2} \frac{L^{2}}{b^{2}}<L^{2}
$$

Since $\tilde{b}^{2}, \tilde{c}^{2}<L^{2}, \tilde{Y} \in \mathcal{A}$. Second, for the inverse mapping, $Y(\tilde{Y} \in \mathcal{A}) \in \mathcal{B}$ is checked as follows:

$$
c^{2}=b^{2}+L^{2}-2 y_{1} L=b^{2}+L^{2}-2 \tilde{y}_{1} \frac{L^{3}}{\tilde{b}^{2}}<b^{2}
$$

since

$$
\tilde{c}^{2}=\tilde{b}^{2}-2 \tilde{y}_{1} L+L^{2}<L^{2} \quad \Leftrightarrow \quad L^{2}-2 \tilde{y}_{1} \frac{L^{3}}{\tilde{b}^{2}}<0
$$

and

$$
b^{2}=L^{2} \frac{L^{2}}{\tilde{b}^{2}}>L^{2}
$$

Since $b>L$ and $b>c, Y \in \mathcal{B}$. Finally, let us look at how the whole integral transforms. First inserting the transformed variables including the Jacobian (B.6) and second using the homogeneity (B.3), we arrive at

$$
\begin{aligned}
\int_{Y \in \mathcal{B}} f(a=L, b, c) & =\int_{\tilde{Y} \in \mathcal{A}}\left(\frac{L}{\tilde{b}}\right)^{2 D} f\left(L, \frac{L^{2}}{\tilde{b}}, \frac{\tilde{c} L}{\tilde{b}}\right) \\
& =\int_{\tilde{Y} \in \mathcal{A}}\left(\frac{L}{\tilde{b}}\right)^{2 D-\lambda} f(\tilde{b}, \tilde{a}=L, \tilde{c}) .
\end{aligned}
$$

Let us restate the above calculation in a completely geometric interpretation as sketched in the figure. The mapping consists of two steps: 
- The rescaling with respect to $\mathrm{O}$ by a factor $L / b$ which maps $Y$ onto $Y^{\prime}$ and $H$ onto $H^{\prime}$. Equation (B.3) implies that $f$ is changed by a factor $\left(\frac{L}{b}\right)^{-\lambda}$.

- A mirror operation, which maps $Y^{\prime}$ onto $H$ and $H^{\prime}$ onto $\tilde{Y}$, leaving invariant the origin $\mathrm{O}$. This operation is a permutation of the first two arguments of $f$.

The mapping $Y \rightarrow \tilde{Y}$ corresponds to the special conformal transformation (B.4).

Analogously, we find that

$$
\int_{Y \in \mathcal{C}} f(a=L, b, c)=\int_{\tilde{Y} \in \mathcal{A}}\left(\frac{L}{\tilde{c}}\right)^{2 D-\lambda} f(\tilde{c}, \tilde{b}, \tilde{a}=L) .
$$

\section{References}

[1] Schäfer L 1999 Excluded Volume Effects in Polymer Solutions (Berlin: Springer)

[2] Blatter G, Feigel'man M V, Geshkenbein V B, Larkin A I and Vinokur V M 1994 Vortices in high-temperature superconductors Rev. Mod. Phys. 661125

[3] Kardar M, Parisi G and Zhang Y-C 1986 Dynamic scaling of growing interfaces Phys. Rev. Lett. 56 889-92

[4] Wiese K J 1999 Polymerized membranes, a review Phase Transitions and Critical Phenomena vol 19 (London: Academic)

[5] Abraham D B 1980 Solvable model with a roughening transition for a planar Ising ferromagnet Phys. Rev. Lett. $441165-8$

[6] Upton P J 1999 Exact interface model for wetting in the planar Ising model Phys. Rev. E 60 3475-8

[7] Nakanishi H and Fisher M E 1982 Multicriticality of wetting, prewetting, and surface transition Phys. Rev. Lett. 49 1565-8

[8] Halperin B I, Brézin E and Leibler S 1983 Critical wetting in three dimensions Phys. Rev. Lett. 501387

[9] Lipowsky R, Kroll D M and Zia R K P 1983 Effective field theory for interface delocalization transitions Phys. Rev. B 27 4499-502

[10] Kroll D M, Lipowsky R and Zia R K P 1985 Universality classes for critical wetting Phys. Rev. B 321862

[11] Fisher D S and Huse D A 1985 Wetting transitions: a functional renormalization-group approach Phys. Rev. B 32 247-56

[12] Lipowsky R and Fisher M E 1987 Scaling regimes and functional renormalization for wetting transitions Phys. Rev. B 36 2126-241

[13] David F and Leibler S 1990 Multicritical unbinding phenomena and nonlinear functional renormalization group Phys. Rev. B 41 12926-9

[14] Forgas G, Lipowsky R and Nieuwenhuizen T M 1991 The behaviour of interfaces in ordered and disordered systems Phase Transitions and Critical Phenomena vol 14 (London: Academic) pp 136-376

[15] Duplantier B 1989 Interaction of crumpled manifolds with Euclidean elements Phys. Rev. Lett. 622337

[16] David F, Duplantier B and Guitter E 1993 Renormalization of crumpled manifolds Phys. Rev. Lett. 702205

[17] David F, Duplantier B and Guitter E 1993 Renormalization theory for interacting crumpled manifolds Nucl. Phys. B 394 555-664

[18] Kantor Y and Nelson D R 1987 Crumpling transition in polymerized membranes Phys. Rev. Lett. 58 2774-7

[19] Kantor Y and Nelson D R 1987 Phase transitions in flexible polymeric surfaces Phys. Rev. A 36 4020-32

[20] Kantor Y, Kardar M and Nelson D R 1986 Statistical mechanics of tethered surfaces Phys. Rev. Lett. 57 791-5

[21] Kantor Y, Kardar M and Nelson D R 1987 Tethered surfaces: statics and dynamics Phys. Rev. A 35 3056-71

[22] Kardar M and Nelson D R $1987 \varepsilon$ expansions for crumpled manifolds Phys. Rev. Lett. 581289 Kardar M and Nelson D R $1987 \varepsilon$ expansions for crumpled manifolds Phys. Rev. Lett. $582280 \mathrm{E}$

[23] Aronovitz J A and Lubensky T C 1988 Fluctuations of solid membranes Phys. Rev. Lett. 60 2634-7

[24] David F, Duplantier B and Guitter E 1994 Renormalization and hyperscaling for self-avoiding manifold models Phys. Rev. Lett. 72311

[25] David F, Duplantier B and Guitter E 1997 Renormalization theory for the self-avoiding polymerized membranes Preprint cond-mat/9702136

[26] David F and Wiese K J 1996 Scaling of self-avoiding tethered membranes: 2-loop renormalization group results Phys. Rev. Lett. 764564

[27] Wiese K J and David F 1997 New renormalization group results for scaling of self-avoiding tethered membranes Nucl. Phys. B 487 529-632

[28] Bowick G, Cacciuto A, Thorleifsson M and Travesset A 2001 Universality classes of self-avoiding fixedconnectivity membranes Eur. Phys. J. E 5149 
[29] Pinnow $\mathrm{H}$ and Wiese K J Work in progress

[30] Wiese K J and David F 1995 Self-avoiding tethered membranes at the tricritical point Nucl. Phys. B 450 $495-557$

[31] Hwa T 1990 Generalized $\varepsilon$ expansion for self-avoiding tethered manifolds Phys. Rev. A 41 1751-6

[32] Lässig M and Lipowsky R 1993 Critical roughening of interfaces: a new class of renormalizable field theories Phys. Rev. Lett. 70 1131-4

[33] Goulian M 1991 The Gaussian approximation for self-avoiding tethered surfaces J. Physique 1 1327-30

[34] Le Doussal P 1992 Tethered membranes with long-range self-avoidance: large dimension limit J. Phys. A: Math. Gen. 25 469-76

[35] Guitter E and Palmeri J 1992 Tethered membranes with long-range interaction Phys. Rev. A 45 734-44

[36] David F and Wiese K J 1998 Large orders for self-avoiding membranes Nucl. Phys. B 535 555-95

[37] Diehl H W 1986 Field-theoretical approach to critical behaviour of surfaces Phase Transitions and Critical Phenomena vol 10 (London: Academic) pp 76-267

[38] Eisenriegler E, Kremer K and Binder K 1982 Adsorption of polymer chains at surfaces: scaling and Monte Carlo analysis J. Chem. Phys. 776296 